\title{
WestVirginiaUniversity
}

THE RESEARCH REPOSITORY @ WVU

Graduate Theses, Dissertations, and Problem Reports

2004

\section{Multicultural competence and levels of effectiveness in adventure -based counseling}

David M. Cummins

West Virginia University

Follow this and additional works at: https://researchrepository.wvu.edu/etd

\section{Recommended Citation}

Cummins, David M., "Multicultural competence and levels of effectiveness in adventure -based counseling" (2004). Graduate Theses, Dissertations, and Problem Reports. 2069.

https://researchrepository.wvu.edu/etd/2069

This Dissertation is protected by copyright and/or related rights. It has been brought to you by the The Research Repository @ WVU with permission from the rights-holder(s). You are free to use this Dissertation in any way that is permitted by the copyright and related rights legislation that applies to your use. For other uses you must obtain permission from the rights-holder(s) directly, unless additional rights are indicated by a Creative Commons license in the record and/ or on the work itself. This Dissertation has been accepted for inclusion in WVU Graduate Theses, Dissertations, and Problem Reports collection by an authorized administrator of The Research Repository @ WVU.

For more information, please contact researchrepository@mail.wvu.edu. 
Multicultural Competence and

Levels of Effectiveness in Adventure Based Counseling

David M. Cummins

Dissertation submitted to the College of Human Resources and Education

at West Virginia University in partial fulfillment of the requirements

for the degree of

Doctor of Philosophy

In

Counseling Psychology

David Srebalus, Ed.D., Chair

Cynthia Kalodner, Ph.D.

Richard Walls, Ph.D.

Samuel Zizzi, Ed.D.

James Delo, Ph.D.

Department of Counseling, Rehabilitation Counseling, and Counseling Psychology

Morgantown, West Virginia

2004

Keywords: Multicultural Competence, Counselor Effectiveness, Adventure Based Counseling, Youth at Risk 


\author{
Abstract \\ Multicultural Competence and \\ Levels of Effectiveness in Adventure Based Counseling
}

David M. Cummins

The current study explored the relationship between adventure based counselor multicultural competency and their levels of effectiveness as perceived by youth at risk. The sample consisted of 22 counselors (19 White and 3 ethnic minority) who competed the Multicultural Knowledge and Awareness Scale and 86 youth at risk participants (52 White and 34 ethnic minority) who completed the Counselor Effectiveness Rating Scale. Analysis of the data suggests a very small negative relationship between multicultural competence of adventure based counselors and their levels of effectiveness as rated by the ethnic minority and White youth at risk participants. Limitations of the study are addressed and recommendations are made for future investigation and the field of adventure therapy. 


\section{ACKNOWLEDGEMENTS}

Completing this document has been a long process full of adventure and misadventure. I would not have accomplished this challenge without the guidance and support of many individuals along the way. First, I'd like to acknowledge and thank my chair, Dave Srebalus, for helping me to see this through. I would also like to acknowledge the rest of my committee for helping me with their expertise and assistance. All of you have played a vital role in helping me to achieve my goals.

I would also like to thank all of the staff at Touch of Nature Environmental Center for their dedication and willingness to help me collect all of my data. I realize the difficulties of the job, and appreciate all of the extra work involved. In particular, I would like to thank Lisa Wait for helping me to see this project through.

I want to thank the faculty at Boise State University for helping me with the statistical analysis. Laura Bond and Roger Stewart gave me the help when I most needed it. I would also like to thank the staff that I work with who have encouraged me along the way and taken steps to provide me the time and resources to complete my study.

I would also like to thank all of my family and friends who have backed me along the way. Thanks for you faith in me, and giving me the love to see this through.

Mahalo! 


\section{TABLE OF CONTENTS}

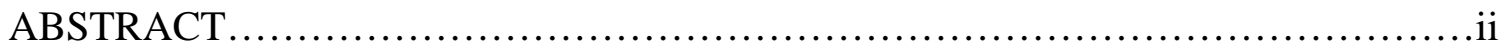

ACKNOWLEDGMENTS..........................................................

CHAPTER 1: Problem in Perspective..........................................1

Introduction......................................................

Statement of the Problem............................................4

Purpose of the Study.............................................4

Research Questions.................................................5

Definition of Terms.............................................5

CHAPTER 2: Literature Review...........................................6

Youth at Risk..................................................6

Youth at Risk Definition....................................8

Research on Treating Youth at Risk...........................9

Adventure Based Treatment.......................................11

History of Adventure Based Treatments.......................12

Adventure Based Treatments: In Search of a Definitions.........15

Adventure Based Interventions with Youth at Risk..............17

Effectiveness of Adventure Based Treatments....................19

Ethical Consideration with Adventure Based Programming......22

Multicultural Competence ......................................26

Ethical Considerations of Multicultural Counseling..............27

Constructs of Multicultural Counseling........................28 
Research on Multicultural Competence.........................30

Comparison of Ethnic Minority and White Multicultural

Competence................................................ 30

White Counselor Multicultural Competence.....................32

General Versus Multicultural Competence........................33

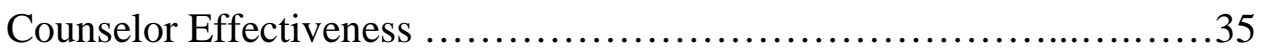

Constructs of Counselor Effectiveness..........................36

Counselor Effectiveness with Ethnic Minorities...................36

Counselor Effectiveness Based on Counselor Ethnic

Similarity/Dissimilarity.....................................37

Counselor Effectiveness Based on Counselor Multicultural

Competence.................................................41

CHAPTER 3: Method......................................................45

Participants.......................................................... 45

Youth at Risk...............................................45

Adventure Based Counselors.....................................46

Treatment Setting................................................... 47

Dependent Measure......................................................

Counselor Effectiveness Rating Scale...........................48

Independent Measures.................................................49

Wilderness Counselor Research Questionnaire..................49

Wilderness Participant Research Questionnaire...................50

Multicultural Knowledge and Awareness Scale...................50 
Research Design........................................................51

Statistical Analysis....................................................51

Procedures............................................................52

CHAPTER 4: Results and Discussion......................................54

Results............................................................54

Data Collection and Demographic Data...............................54

Counselor Effectiveness Rating Scale Analysis......................56

Multicultural Counseling Knowledge and Awareness Scale Analysis.....64

Counselor Effectiveness and Multicultural Competence Analysis........66

Discussion........................................................... 70

Summary of Results and Relation to Previous Research.................70

Counselor Effectiveness..................................70

Counselor Multicultural Competence...........................73

Relation Between Counselor Effectiveness and Multicultural

Competence..............................................74

Limitations of the Study.......................................74

Recommendations for Future Research............................77

Recommendations for the Field of Adventure Based Programming......80

CHAPTER 5: Summary...............................................85

Youth at Risk.....................................................85

Adventure Based Treatment.......................................86

Multicultural Competence........................................89

Counselor Effectiveness.............................................91 
Selection of Participants...........................................992

Instruments...................................................92

Procedures.........................................................93

Results......................................................94

Discussion......................................................95

REFERENCES........................................................97

APPENDIX A............................................................ 114

Description of the Thirty Day Wilderness Therapy Program

APPENDIX B....................................................119

Theoretical Underpinnings of the Wilderness Therapy Program

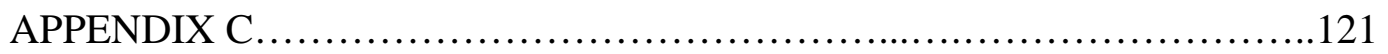

Wilderness Participant Research Questionnaire

APPENDIX D........................................................ 122

Wilderness Questionnaire Script and Administration Directions

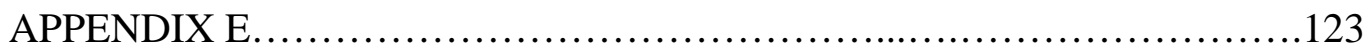

Wilderness Counselor Research Questionnaire 


\author{
Multicultural Competence and \\ Levels of Effectiveness in Adventure Based Counseling \\ CHAPTER ONE \\ Problem in Perspective
}

\title{
Introduction
}

As the population of Youth at risk continues to rise, so does the need for effective forms of treatment (Fields \& MacNamara, 2003). Today’s youth face numerous stressors on a daily basis, and many of them attempt to cope with these stressors in negative ways (McWhirter, McWhirter, McWhirter, \& McWhirter, 1993). Many minority youths have a higher exposure to risk factors, which results in an even higher prevalence of delinquent and self-destructive behaviors (Harvey \& Coleman, 1997; Stevens \& Griffin, 2001). Many ethnic minority youth attempt to cope with the problems of substandard environments and prejudice in negative ways, and may manifest themselves as substance abuse, criminal behavior, sexual activity, and psychological disorders (Noguera, 2003; Poussaint, 2000). Despite the utilization of various forms of treatment, few have shown significant promise, and the population of at risk youth continues to grow at an alarming rate (Davis, 1999). One form of treatment, adventure based counseling, has shown some merit as a viable option to incarceration and traditional forms of treatment (Buyer, 2002; Davis-Berman \& Berman, 1994).

Adventure based treatment for youth at risk is a form of intervention that concentrates on experiential-based learning to facilitate behavior change in a wilderness setting (Gass, 1993). These programs create experiences that challenge teens to think and 
behave in novel and healthier ways (Kraft \& Sakofs, 1985), encourage problem solving and critical thinking (Kraft \& Sakofs, 1985), increases awareness of one’s constructive as well as maladaptive patterns through direct experience (Gass, 1993), and present strong reinforcement properties and accountability through immediate and natural consequences (Kraft and Sakofs, 1985). Over the past 30 years, adventure based counseling has grown from a handful of programs to over 300 in the United States (Bruyere, 2002). Most of these programs concentrate on treating youths who display and experience various forms of risk (Davis-Berman \& Berman, 1994). A large proportion of the youths served by these programs are ethnic minorities from economically impoverished environments, while the vast majority of the counselors tend to be White and from middleclass homes (Benepe, 1992; Warren, 2002). In light of this discrepancy, the field has done little to address the need for adventure based counselors to competently and effectively treat diverse populations (Warren, 1999).

There has been substantial growth in understanding the importance of multicultural issues in psychology and other human service fields over the past 30 years (Atkinson, Morten, \& Sue, 1993; Mio \& Iwamasa, 1993). In order to better understand multicultural counseling, Sue, Bernier, Durran, Feinberg, Pedersen, Smith, and VazquezNutall (1982) wrote an influential paper calling for the development of multicultural competencies. Sue et al. (1982) identified 11 competencies that are generally considered to be the minimum standard for training, research, and practice in counseling (Arredondo, Toporek, Brown, Jones, Locke, Sanchez, and Stadler, 1996). These 11 competencies were later refined into three basic constructs: awareness, knowledge, and skill (Sue, Arrendondo, \& McDavis, 1992). 
Even in light of this progress, research on multicultural competence and counseling continues to be under-represented in social science literature. The main force behind the need for more multicultural competence is from the realization that the field of psychology has tended to inadequately attempted to address culturally complex issues with culture-blind theory based predominantly on European American contexts (Atkinson, Morten, \& Sue, 1993). Fortunately, the trend for more multicultural understanding has branched out to many other professions, including the adventure based counseling field (Warren, 2002). However, as with many other social service fields, the discipline of adventured based counseling has neglected to adequately explore this area of concern.

One method of understanding multicultural competence is by exploring the impact that it has on the consumer's perception of counselor effectiveness (Fuertes \& Brobst, 2002). Investigation in this area is particularly important because ethnic minority mental health needs have not been adequately met in application nor in research (Okonji \& Osokie, 1996; Ridley, 1989). Most of the literature exploring multicultural issues and levels of counselor effectiveness is based on Sue, et al.'s (1992) three constructs of multicultural competence (i.e. awareness, knowledge, and skill) and Strong’s (1968) assessment of counselor characteristics and client satisfaction (i.e. expertness, attractiveness, and trustworthiness) (Constantine, 2002; Fuertes, 2001).

The current study investigates the relationship between the self-reported multicultural competence of adventure based counselor and their levels of effectiveness as rated by the youth at risk participants. Investigating the impact that adventure based counselor multicultural competence and the relation it has to their effectiveness ratings by 
ethnic minority and White youth at risk are important steps in furthering these bodies of research as well as taking steps to improve practice in related areas.

\section{Statement of the Problem}

There appears to be general consensus within the bodies of literature exploring youth at risk ethnicity, adventure based treatment, counselor effectiveness, and multicultural competence that additional research as well as improved theory and application is needed. Research related to youth at risk tends to suggest that traditional forms of treatment are not as effective as originally thought, and more effective means of treatment need to be identified and applied (Bruyere, 2002). One area that has displayed consistent effectiveness with youth at risk is adventure based treatment programs (Bruyere, 2002). However, little literature and research was found that addressed adventure based treatment based on ethnicity. One means of attaining a better direction is by determining which factors impact ethnic minority and White youth at risk levels of perceived effectiveness with their counselors, in regards to their levels of multicultural competence. Literature indicates that ethnically similar and multiculturally competent counselors are deemed more effective by their ethnic minority clients. This study addresses the impact adventure based counselors have on their ethnic minority and White youth at risk clientele by exploring the relationship that their levels multicultural competence have on their perceived levels of effectiveness.

\section{Purpose of the Study}

The purpose of this study was to investigate the impact that adventure based counselor levels of multicultural competence have on their effectiveness ratings by ethnic minority and White youth at risk participants. This area of research is particularly 
valuable because of the lack of methodologically strong empirical research on adventure based programming and the absence of literature that investigates multicultural competencies and counselor effectiveness in the field of adventure based treatment.

\section{Research Questions}

1. How does multicultural competence of adventure based counselors relate to ethnic minority and White participant ratings of their counseling effectiveness?

2. Are the generated results of Research Question 1 due to multicultural counseling competence or are they the result of general counseling competence?

\section{Definition of Terms}

Youth at Risk: Youth at risk are adolescents who have demonstrated some behavioral and/or emotional problems that are severely affecting his or her relationship with family, school, or community.

Adventure Based Treatment: Adventure based treatment utilizes various experiential based interventions in a wilderness context for therapeutic purposes.

Multicultural Competence: Multicultural Competence refers to a counselor's ability to effectively integrate multicultural and culture specific Awareness, Skills, and Knowledge in a counseling context.

Counselor Effectiveness: Counselor Effectiveness refers to a facilitator's credibility and efficacy, in relation to the counselor's Attractiveness, Expertness, and Trustworthiness as perceived by the population that he or she is serving.

Ethnic Minority: Ethnic Minority refers to five major cultural groups: African/Black, Asian, Hispanic/Latino, Native American, and Bi-racial. 


\section{CHAPTER TWO}

\section{Review of Relevant Literature}

This chapter reviews the relevant literature related to multicultural competence and counselor effectiveness ratings with adventure based treatment for youth at risk. First, is a review of the current status of at risk youth and forms of treatment. Second, is a review of adventure based treatment. Third, is a review of counselor multicultural competence. And finally, is a review of counselor effectiveness with ethnic minorities. Youth at Risk

Our society has a significant investment in the health and well being of today’s adolescents for the sake of the youth, as well as that of America's future. The rates of destructive and unhealthy behavior for youths in America have shown dramatic increase over the past two decades, and are currently at an alarming rate (Fields \& McNamara, 2003). Not only is the frequency increasing, but today's youth are participating in risky behaviors at a younger and younger age (Stevens \& Grifin, 2001). Some of these include increases in homicide (victim and perpetrator), drug use, acts of violence, incarceration, HIV, and suicide (Franke, 2000; Noguera, 2003, Poussaint, 2000). Compared to White youth, ethnic minority youth participate in an even higher prevalence of activities and situations that are dangerous to their health and safety as well as that of others (Franke, 2000; Harvey \& Coleman, 1997; Stevens \&Griffin, 2001).

According to Franke (2000), an overwhelming number of today’s ethnic minority youth grow up in particularly impoverished and dangerous conditions that promote highrisk behaviors. Because ethnic minority adolescents are oftentimes subjected to exploitation, discrimination, and subordination by White society, in addition to the other 
pressures of adolescence, they have been referred to as "outcasts among immigrants" (Howard-Hamilton \& Behar-Horenstein, 1995). Challenges that many of these youth face, such as growing up in poverty, single parent homes, facing internal as well as external discrimination, negative societal and media messages, and severely limited opportunities negatively impact their sense of self, others, and the world (Davis, 1999). These inequalities in economics and social justice lead to dissatisfaction and frustration in ethnic minority youth and directly contribute to their higher prevalence of antisocial and criminal behavior (Gullotta, 2000). This mixture of risk factors coupled with peer pressure and a need for identity creates a ,e for unhealthy and destructive responses for many ethnic minority youth (Gullotta, 2000). Tidwell and Garrett (1994) note that these behaviors and attitudes develop into a cumulative, self-fulfilling cycle of deviance. Many of the "negative" behaviors youth at risk display are actually attempts at coping with their daily struggles inherent in their socio-cultural environment (McWhirter, McWhirter, McWhirter, \& McWhirter, 1993). The combination of these factors combined with a lack of resources, ethnic minority adolescents are at a high disposition to utilize coping strategies that have negative and destructive consequences (Howard-Hamilton \& BeharHorenstein, 1995,).

In a system that is "fixed" against ethnic minorities, these youth learn to manipulate it by

(a) collective struggle; the failure that results from a system that does not afford equal opportunity to all of its members; therefore, individuals abdicate responsibility for their faults; 
(b) clientship; rewards for task completion that are perceived to be contingent on one's ability to successfully manipulate those in power; and

(c) hustling; material success and prestige achieved by exploiting and manipulating others (Howard-Hamilton \& BeharHorenstein, 1995, p. 199).

Eventually, these survival skills, negative adaptive strategies, and search for meaning develop into destructive behavioral patterns (Capuzzi \& Gross, 1996). With the various stressors that ethnic minorities face, it is not surprising that sex, drug and alcohol abuse, violence, disregard for others, and crime are oftentimes attempts at controlling and coping with these substandard, unhealthy, and discriminatory environments.

Youth at Risk Definition. Despite the vast amount of media attention to this topic and negative impact on society, empirical and theoretical deficiencies exist in this body of literature (McWhirter, McWhirter, McWhirter, \& McWhirter, 1993). One limitation has been a lack of a consistent definition for "youth at risk". The definition of "youth at risk" has long been debated, and Tidwell and Garrett (1994) discuss the difficulties and pitfalls of defining youth at risk. The authors note that it is a very broad issue, and that a universally accepted definition of the term has yet to be accepted. The definition to be used in this study is based on literature from the youth at risk program utilized in the current research. According to their literature, youth at risk are adolescents who verge of serious trouble in school, at home, in the community, or in traditional placements. These youths have demonstrated some behavioral and/or emotional problems that are severely affecting his or her relationship with family, school, or community. These problems 
include, but are not limited to, physically aggressive behavior, severe truancy, juvenile court contact, runaway behavior, and drug abuse (Spectrum Wilderness Programs, 2004). Research on Treating Youth at Risk. Considering the breadth of this problem, there is a noticeable lack of solid empirical literature on treating youth at risk. According to Dalton (1998) some solid research exists, but "their overall dearth in numbers was noteworthy” (p. 1083). Even with these theoretical and empirical limitations, many forms of treatment have been instituted to manage the problems that youth face as well as the problems they cause in society. A number of these programs and forms of interventions have been developed over the past decades to assist and treat youth at risk, including incarceration, probation, boot camps, talk therapies, alternative schools, community service, rehabilitation programs, and mentoring programs (Dryfoos, 1990; McWhirter, McWhirter, McWhirter, \& McWhirter, 1993). These organizations use a variety of interventions in hopes of helping youth; including punishment, peer support, confrontation, education, and rehabilitation (Albanese, 1993). Some of the "traditional" forms of treatment have demonstrated to not be as effective as originally thought. Bruyere (2002) suggests that "traditional and autocratic approaches, such as individual therapy, are often not successful interventions for delinquent youth” (p. 3). Studies have supported this by suggesting that established, punitive, and authoritarian styles are not effective forms of intervention with delinquent youth (Stutzner-Gibson, Koren, \& DeChillo, 1995). This indicates that alternative interventions need to be investigated, developed, and employed (Bazemore \& Clinton, 1997).

Research has found that rehabilitation (Boone, 1994), group counseling (Goodman \& Getzbel, 1996; Zink \& Littrell, 2000), and interventions based on action 
and healthy risk (Albanese, 1993) are particularly effective at reducing the level of risk with adolescents. Adventure based interventions are an alternative approach to serving youth at risk that typically employs rehabilitation, group counseling, and action has demonstrated particular merit (Buyere, 2002; Davis-Berman \& Berman, 1994).

A theoretical and practical shortcoming to treating youth at risk is that little attention has been given to investigating cultural issues related to causes, prevention, and treatment with ethnic minorities (Liu \& Clay, 2002). Harvey and Coleman (1997) support this with their claim that "most service in the juvenile justice system have no culturally relevant interventions directed toward changing negative behaviors among high-risk youth” (p. 197). Adventure based programs have these shortcomings as well. Although no study on the percentages of ethnic minority participation in adventure therapy programs could be found, it is likely that the disproportionate percentage of ethnic minority youth in our society suggests a high representation in voluntary and nonvoluntary participants. According to Bruyere (2002), delinquent youth adventure based programs "are very diverse”. Consequently, the available interventions must be equally diverse to accommodate the expected demographic, developmental and character differences” (p. 207). Being able to more effectively treat ethnically diverse youth at risk in adventure based programs would have potentially significant benefit for the youths as well as society.

As with all schools of thought based primarily by White culture and values, adventure based theory and practice does not adequately meet the culture-specific needs of ethnic minority youth at risk (Warren, 2002). It is imperative that the counselors and administrators of these program serving ethnic minority adolescents understand and 
intervene with their clients of color from a cultural standpoint (Howard-Hamilton \& Behar-Horenstein, 1995). These youths are in need of counselors who can adequately comprehend the challenges they face, can effectively communicate with them, can see the world more closely through their eyes, and understand how they relate to it. One approach towards gaining insight into this neglected area of research is by investigating the ethnic minority and White participants perceptions of their counselors as related to their multicultural competence (Buyere, 2002; Warren, 2002).

\section{Adventure Based Treatment}

Adventure based counseling, a form of intervention that concentrates on experiential-based learning to facilitate behavior change in the wilderness (Gass, 1993), is one manner of intervention that has shown merit and substantial development in treating youth at risk (Bandoroff, 1992). In the past 30 years, programs that utilized adventure based interventions have grown in the United States from a handful to numbers ranging in the hundreds (Bruyere, 2002). Although relatively new at serving youth at risk, adventure based approaches have been used to help teens learn, grow, and improve their lives since the 1940s (Davis-Berman \& Berman, 1994).

Adventure based treatment's historical and theoretical foundations are established on the concepts of experiential education. Adventure based programming tends to based primarily on the concept that behavior change and deeper learning happen more effectively through direct experience (Kraft \& Sakofs, 1985). Kraft and Sakofs’ experiential concepts apply to adventure based interventions by:

1. The learner is participant rather than spectator in learning. 
2. The learning activities requite personal motivation in the form of energy, involvement, and responsibility.

3. The learning activity is real and meaningful in terms of natural consequences for the learner.

4. Reflection is a critical element the learning process.

5. Learning must have present as well as future relevance for the learner and the society in which he or she is a member (Gass, 1992, p.4)

Adventure based treatment programs typically draw from these concepts in several ways. They create experiences that challenges persons to think and behave in novel and healthier ways (Kraft and Sakofs, 1985), encourages problem solving and critical thinking (Herbert, 1996), increases awareness of one’s constructive as well as maladaptive patterns through direct experience (Gass, 1993), presents strong reinforcement properties and accountability through immediate and natural consequences (Russell, 2001), and motivates through the use of fun (Herbert, 1996).

History of Adventure Based Treatments. Some of the early contributors to experiential education include Plato, Aristotle, John Dewey, William James, and Kurt Hahn (Herbert, 1996). Arguably the most influential person in the development of adventure as a means of treatment and personal growth was Kurt Hahn (Miner \& Boldt, 1981). As early as the 1930s, Hahn utilized experiential education concepts as a means for personal growth and change (James, 1980). Hahn created the first Outward Bound school (OB), after observing that younger and stronger sailors in World War II were dying more rapidly than older more experienced sailors while under the same conditions 
of extreme stress and trauma (Davis-Berman \& Berman, 1994). From this observation, Hahn concluded one's mental faculties were just as important as one's physical fitness in survival (Miner \& Boldt, 1981). In 1941 Hahn opened the first OB school and used his educational philosophies, wilderness and rescue training, as well as social cooperation to make the younger seamen more resilient to the demands of war and seamanship. Hahn later generalized these concepts and applied this philosophy to the general public through OB (Miner \& Boldt, 1981).

OB opened its first U. S. branches in the early 1960s and by the 1980 there were hundreds of programs using OB based approaches in various forms and environments (Bacon \& Kimball, 1989; Davis-Berman \& Davis, 1994). These programs vary in their philosophies and approaches, but they all have the basic foundation as outlined by Hahn: “To serve, to strive, and not to yield” (James, 1980). In the 1970s, health professionals began to appreciate the therapeutic value of the OB approach and began to apply it to groups of people in need of psychological assistance; particularly troubled youth. Hahn’s philosophy was thus transformed from preparing “young British seamen to survive the rigors of sailing the North Atlantic during World War II” (Bacon \& Kimball, 1989, p. 117) into present day adventure based treatment where the goal is to prepare young clients to survive the rigors of life. OB programs regularly began to include adjudicated youths in their programs; and early studies found support that Hahn’s approach was effective in significantly reducing delinquent behaviors (Bacon \& Kimball, 1989; DavisBerman \& Berman, 1994).

The OB process is especially suited for treating youth at risk because: 
1. The tasks are inherently concrete, manageable, and solvable within a limited timeframe.

2. Mastery of the skills provides participants with a sense of accomplishment. It builds on one's strengths and reinforces positive aspect rather than highlighting failures and weaknesses. In this sense it focuses on the healthier, functioning part of the individual. The complexity and difficulty of the challenges corresponds to incremental levels of instruction. By performing a needs assessment of the group, the instructor determines the starting-off point and developmental sequence of the activities, which increases the probability of success.

3. The supportive climate created by the instructor and peer group as well as the interaction generated by addressing specific tasks, allows conflicts, anxieties, and difficulties to deal with openly and examined in a straight forward manner. (Stitch \& Gaylor, 1983, p. 3)

The program used in the present study consulted with OB, because of its success with troubled teens, during its creation in the mid-1970s. The program based many of its theoretical and philosophical underpinning on the OB process; and the program continues to employ many of these as the basis for its interventions (Spectrum Wilderness Programs, 2004; Wichmann, 1991). 
Adventure Based Treatments: In Search of a Definitions. Many different programs with varying philosophies and forms of interventions have been developed since the days that OB first opened its doors. This has allowed for significant development in the field with varied views and approaches to treating youth at risk (Davis-Berman \& Berman, 1994). However, inconsistency in terms and definitions related to interventions that take place in a wilderness context or have an adventure-based component has been a problem for the profession and related research (Gillis, 1995). For example, it is common for professionals to use the terms adventure based therapy and wilderness therapy interchangeably to describe the same form of intervention (Gillis, 1995). The findings of Davis-Berman, Berman, and Capone (1994) also suggest the profession, as a whole has neither reached an agreement on what constitutes adventure based treatment programs nor an operational definition for it. One of the difficulties in trying to define adventure based counseling is due to the wide variety of organizations and agencies that employ adventure based programming in various settings with diverse populations (Russell, 2001). In an attempt to secure a definition, professionals within the Therapeutic Adventure Professional Group (TAPG), the leading professional group in the adventure counseling profession, have been involved in an ongoing debate as to what constitutes adventure and wilderness counseling. In one article, Gillis (1995) defined "adventure therapy as an active approach to psychotherapy for people seeking behavioral change, either voluntary or through some court ordered coercion, that utilizes adventure activities, be they group games and initiatives or wilderness expeditions (with some real or perceived risk) as the primary therapeutic medium” (p. 6). 
Although not a clinical program, the philosophy, intervention strategies, and setup of the program used in the current study most closely resembles the definition of “wilderness therapy” as outlined by Gass (1993). Wilderness therapy, as defined by Gass, takes place when a "therapeutic experience occurs in remote wilderness settings and tends to consist of very small groups (8-15), multiple days (e.g., 24 days), and round the clock interventions” (p. 9).

Adventure Based Interventions with Youth at Risk. Although youth at risk are not the only population that participates in programs that use adventure as a means of treatment, they do comprise the largest portion of the populations served (Davis-Berman \& Berman, 1993). The main goals for most of these programs are to help at risk youth replace selfdestructive behaviors with healthier ones (Gass, 1993). A significant body of research exists that indicates that this type of intervention is an effective form of treatment for many issues that youth at risk present (Bruyere, 2002). These programs oftentimes attempt to do this by providing challenging opportunities for growth, increasing prosocial values, improving decision making skills, and by decreasing helplessness and passivity (Gass, 1993; Bacon \& Kimball, 1989). Among many other problems that youth at risk face, studies have illustrated that adventure based programming has been effective with at risk youth in treating substance abuse (Gillis \& Simpson, 1991; Skafos, 1992), reducing recidivism rates (Newes, 2001; Sakofs, 1992; Wichmann, 1991), enhancing interpersonal skills (Herbert, 1996), treating mental illnesses (Kelley \& Selby, 1997), improving self-esteem (Moote \& Wodarski, 1997), and improving locus of control (Han, 2000). 
Adventure based programming offers unique conditions and situations that make it particularly effective with youth at risk. Many of the programs take the youths out of unhealthy environments and put them into a wilderness setting where their past interaction styles become ineffective (Gass, 1993). This novel environment creates a situation where the youths have to think in new ways that help them gain healthier approaches to getting their needs met as well gaining a different perspective on life (Gass, 1993; Herbert, 1996).

The wilderness provides many opportunities for natural consequences, both positive and negative. Frequently, behaviors have direct consequences and the youths are reinforced immediately for their good and poor decision making. When living in a wilderness setting and dealing with Mother Nature they find that they have no one to accept responsibility but themselves (Davis- Berman \& Berman, 1994; Gass, 1993). Fletcher and Hinkle (2002) claim that the conditions and adventure based interventions allow youths the opportunity to change dysfunctional patterns of behavior and to redefine themselves. The authors note that old destructive patterns of behavior tend to not be reinforced, so participants adapt by learning and applying behaviors that tend to be more constructive and pro-social

Not only does living in the wilderness presents many challenges (Gass, 1993), but adventure based programs are often devised to present progressive stressors and challenges to the youths (Davis-Berman \& Berman, 1994). Presenting the challenges in a progressive manner sets the youths up for success and helps them build a sense of accomplishment, improve self-esteem, understand the benefits of teamwork, and provide 
an opportunity for growth (Bacon \& Kimball, 1989; Davis-Berman \& Berman, 1994, Gass, 1993).

Another common concept in adventure based program is assisting the participants to “push their comfort zone” (Fletcher \& Hinkle, 2002). These progressive challenges usually put the youths in stressful situations that sometimes entail perceived and actual risk. Davis-Berman \& Berman (1994) contend that some of the most significant growth occurs when participants are on the edge or go slightly beyond their comfort zone. When youths successfully do this, they typically are proud of their accomplishments, their comfort zone expands, and they become richer individuals. This growth can help increase the level of the youth at risks resiliency to environmental stressors and make them less susceptible to giving in to unhealthy patterns and influences (Davis-Berman \& Berman, 1994). Many programs utilize debriefing and processing techniques that helps the youths understand how these experiences and growth can be applied in other areas of their lives (Gass, 1999; Rosol, 2000).

These challenges also present the opportunity for conflict to arise. During times of stress, many of the youths' dysfunctional patterns emerge which allows the adventure based counselors and the youths' peers to work directly with many of the issues that have been putting the youths at risk (Wichmann, 1991). Most of these programs are devised to help improve communication, cooperation, and problem solving by presenting and debriefing challenging situations. Adventure based programs use various techniques for individual and group problem solving. Many programs utilize “circles,” a form of group problem solving, when these conflicts or problems present themselves (Wichmann, 
1991). The concept of circles is discussed in more detail in the methods section of this study.

Effectiveness of Adventure Based Treatments. Although the body of research for adventure based programming is based on various populations, the vast majority of it has been conducted on troubled teenagers (Friese, Pittman, \& Hendee, 1995). Literature on adventure based programming as a whole claims to have an overwhelming success with few negative outcomes in treating youth at risk (Burton, 1981; Friese, Pittman, \& Hendee, 1995).

Bandoroff (1992) conducted a considerable review of literature on adventure based programs serving delinquent and pre-delinquent adolescents. As a whole, Bandoroff concluded the studies tended to report lower recidivism rates, higher sense of inclusion, increased positive social attitudes and adjustment, as well as more realistic self-perceptions. Although not based on rigorous statistical analysis, Bandoroff concluded that "the field of adventure programming has succeeded in justifying itself as a viable therapeutic intervention for delinquent youth.” (p. 55).

Interest in empirical support for the efficacy of adventure based treatment programs for troubled teens has been growing over the past decades and recently there has been increasing pressure within the field to produce higher quality research (DavisBerman, Berman, \& Capone, 1994; Neill, 2003; Newes, 2001). Although a number of meta-analyses have demonstrated adventure based programming as an effective mode of treatment for various identified populations, they have also illuminated many of the methodological and experimental problems that exist within the body of literature (Cason \& Gillis, 1994; Han, 2000; Hattie, Marsh, Neill, \& Richards, 1997). 
Cason and Gillis’ (1994) conducted a meta-analysis of adventure-based studies with adolescents. Of the original 79 acquired studies, only 43 were included because of methodological weaknesses in the remaining 36 studies. Eighty-eight of the original 235 effect sizes were also excluded due to various methodological issues. The quality of the remaining studies was ranked on a scale from 1 to 6 in regards to research designs and methodological soundness. Of the 43 studies included in the meta-analyses, only five studies achieved a rank of five or higher. Analyzing the 147 effect sizes, from the 43 studies, resulted in a mean effect size of .314 and ranged from -1.48 to 4.26 with a standard deviation of .62. The researchers acknowledged that wide range in effect sizes made assessing the effectiveness of the results less reliable. With this taken into consideration the mean effect size signifies a $12 \%$ improvement, which indicates $62.2 \%$ of the participants who participated were "better off" than those who did not participate. Cason \& Gillis also noted that the most consistent findings in the literature suggest adventure-based programs have a positive effect on self-concept and that longer programs with younger participants were linked with larger effect sizes. However, the authors noted that even though their findings were positive, they only found limited support for adventure programming effectiveness. The authors acknowledged that, although they found an effect, .314 is considered to be very small to moderate for an effect size.

Han (2000) utilized meta-analysis to investigate the effect of adventure based programming on locus of control for 24 studies on adolescents, including 30 effect sizes. Similar to the effect size in Cason and Gillis (1994), Han determined a summary effect size of .38 that indicated an increase in internal control with only two of the 24 studies 
having negative outcomes. Further analysis indicated that programs with a goal of primary therapy had a significantly higher summary effect size (.64) than those with goals of education (.38), development or prevention (.35), and adjunctive therapy (.30). Han suggested this might be interpreted as program goals directly influencing adventure based outcomes. One consideration with the article is Han's failure to establish rigorous criteria for inclusion in the meta-analysis. It is likely that this resulted in the inclusion of some studies with methodological weaknesses.

A third meta-analyses, Hattie et al. (1997) analyzed the effect sizes of 96 studies conducted on Outward Bound programming. Although all Outward Bound programs tended to have some form of therapeutic value, not all of the studies included were therapeutically oriented. Hattie et al. produced an overall summary effect size of .34 with a summary effect size of .51 for post-participation assessment of up to 18 months with a mean of 5.5 months. The authors believe that this indicates that participants continue to experience growth and change after returning to their home environments.

In his review of three meta-analyses of adventure based program outcomes (Cason \& Gillis, 1994; Hattie et al., 1997), Neill (2003) concluded that the collective outcome of the meta-analyses suggest very small to medium outcomes with considerable variability between effect sizes. He reported that the overall effects appear to be positive and ongoing, with an increase of effectiveness with older age of participants and longer duration of programming. However, the effect sizes for adventure based programming (.3 to .4) are still low in comparison to in individual psychotherapy (.68) (Smith \& Glass, 1977). 
Even though the literature on adventure based program effectiveness serving adolescents have resulted in encouraging directions, the literature as a whole appears to be riddled with weaknesses (Newes, 2001). This is primarily due to methodological shortcomings, lack of scientific rigor, and low level of empirical analysis (Chambless \& Hollon, 1998; Gillis, 1992, Newes, 2001). Numerous studies in this field even fail to utilize basic experimental controls, such as random assignment and comparison groups (Moote and Wordarski, 1997; Newes, 2001). Further, most studies have been reported in non-peer reviewed professional journals, and much of the theory is based on anecdotal descriptions and speculation rather than empirical validation (Davis-Berman, Berman, \& Capone, 1994, Neill, 2003). All of this suggests a necessity for more solid research to be conducted in the field of adventure based programming with adolescents.

A particular area that appears to have been neglected within the field is accessing the characteristics of the facilitators and the impact that it has on the clients. Weston, Tinsley, and O’Dell (1999) suggested that future research in adventure based programming attend more to the qualifications and characteristics of counselors. No research could be found that addresses this issue in relation to multicultural competency of the counselors.

Ethical Consideration with Adventure Based Programming. An area of concern with programs in the field of adventure based treatment is that they typically do not have empirically validated theoretical foundations to base their interventions on nor do they consistently implement established theory in their programming (Matthews, 1991; Newes, 2001). Gass (1993) addressed this shortcoming stating that professionals in the field of adventure treatment and programs that employ adventure based interventions 
should have a solid foundation in psychology and its applications. However, a survey of 31 wilderness programs specializing in mental health treatment found very few of the programs they contacted could identify what type of process or therapy they were utilizing with their clients (Gass, 1993).

What is further troubling is that these inconsistently applied theories and techniques are predominantly based on theoretical models and philosophies that do not take into account issues related to non-White races, gender, and social classes (Warren, 1999). Warren (1999) points out that the roots of experiential education are founded on the power dominance of the white male, class privileged, Euro-American belief system. As a result, well intentioned practitioners adopt and apply “culture-blind” and "one size fits all” methods without critically determining their appropriateness for diverse groups. The assumptions that these programs are based on risk, challenge, servitude, and individual accomplishment may not be relevant nor effective with diverse cultures, women, or other marginalized people (Warren, 1999). For instance it may be ineffective as well as potentially endangering to teach an African American youth from a gang in inner-city Chicago White-based values, such as assertiveness skills. Consequently, Warren implies that, ethically and practically, the field of adventure based programming currently is not adequately suited to treat and meet the needs of culturally diverse individuals.

As early as 1979, the Association of Experiential Education took steps to address issues related to cultural diversity (Kielsmeier, 1989); and in 1992 the Therapeutic Adventure Professional Group (TAPG) developed a set of ethical guidelines that addresses some of these concerns. In creating these ethical standards, the TAPG 
consulted with the American Psychological Association (APA), the American

Association of Marriage and Family Therapists (AAMFT), and numerous experts in the field of therapeutic adventure; as well as utilizing influential articles that had been written on related areas (Therapeutic Adventure Professional Group, 2002).

Although similar to the APA ethics code, Hunt (1994) and Mitten (1994) stress it is necessary to go beyond the principles set forth by the APA in order to address issues that are specific to the therapeutic adventure field. Particular to this study, are the principles that address issues related to serving ethnic minorities. A number of the TAPG ethical principles directly or indirectly address issues that pertain to providing or not providing services to ethnic minorities in an ethical manner (Therapeutic Adventure Professional Group, 2002).

The first principle that relates to ethnic minorities is Boundaries of Confidence. This requires TAPG practitioners to provide services only within the boundaries of their experience, education, training, and appropriate supervision; to take necessary steps to ensure that they provide competent research and intervention; to remain current with information in the field; and to maintain current knowledge, practice, and skills (Therapeutic Adventure Professional Group, 2002). The second ethical principle that relates to ethnic minorities is Professional Responsibility. Mitten (1994) and Warren (2002) stresses that TAPG practitioners need to adapt their intervention strategies and methods of treatment to the particular needs of diverse populations, as well as possess adequate knowledge and experience to make appropriate professional judgments with these populations. The third ethical principle that relates to ethnic minorities is Respect for People's Rights and Dignity. Not only does this principle assert that TAPG 
practitioners need to be sensitive to cultural issues and to recognize individual differences of the participants, but TAPG practitioners also do not discriminate on race or ethnicity. The fourth ethical principle that relates to ethnic minorities is Concern for Welfare. TAPG practitioners are to be sensitive to real and ascribed differences in power between themselves and their participants. This applies to working with ethnic minorities due to discrimination and the imbalance of power found between the ethnicities. And, the final principle that relates to ethnic minorities is Social Responsibility that states TAPG staff should recognize their level of social responsibility. This principle stresses TAPG facilitators need to be aware of societal issues that impact their profession as well as their responsibility to social injustices, such as discrimination and prejudice. Hunt (1994) supports this by stressing that TAPG facilitators should remain aware of the social implications of their work and their actions as well as the social differences that exist between them and their participants (Therapeutic Adventure Professional Group, 2002).

Very small steps have been taken towards increasing multicultural competence in the field of adventure based treatment (Kielsmeier, 1989). These steps have brought up enlightening issues and discussion, but they have tended to be shortsighted and anecdotal in nature (Warren, 1999). In her content analysis of outdoor leadership texts, Warren (2002) stress that the field of adventure programming needs to continue expanding research and training in order to accomplish the ethical guidelines for working with its diverse clientele set by the TAPG.

\section{Multicultural Competence}

Since its 1974 Vail conference, the American Psychological Association has increased efforts on ethically treating issues of diversity and increasing multicultural 
competency in counseling (Atkinson, Morten, \& Sue, 1993). Mio and Iwamasa (1993) refer to this interest and growth as an "explosive increase" in multicultural investigation. The main force behind more multicultural competence was from the realization that the field of psychology had inadequately attempted to address culturally complex issues with culture-blind theory based predominantly on European American contexts (Atkinson et al., 1993). Pedersen (1991) claim that ethnic minority clients are likely to be better served by theoretical approaches and culturally sensitive interventions that are created on constructs more similar to their value systems than that typically employed in traditional forms of intervention. In his article, Pederson (1991) contends that multiculturalism is an essential and distinct component of effective counseling. He believes that to be effectual, counselors must go beyond general counseling skills by valuing and recognizing the impact that cultural influences have on the client and counselor as well as in their relationship. The competent counselor should not only be able to apply general counseling skills, but to also adapt interventions, assessment, theoretical interpretations, and techniques to accurately understand clients and address needs specific to his or her culture (Pope-Davis, Liu, Toporek, \& Brittan-Powell, 2001).

Fortunately, the trend for more multicultural understanding and application has branched out to many other professions. In particular, various human service fields that work with youth at risk, such as outdoor education (Roberts \& Rodriguez, 1999), teaching (Baruth \& Manning, 2000; Bennet, 1995; Gomez, 1991), school counseling (Holcomb-McCoy, 2001) corrections (Myers, 2000), law enforcement (Block, 1994; Youngs \& Novas, 1995), the juvenile justice system (Harvey \& Coleman, 1997), and adventure based programming (Warren, 2003) have identified the importance of 
multicultural competence and have taken steps toward training, empirical validation, and setting professional standards. However, Casas and Miguel (1993) voice some caution and disagreement to these optimistic assumptions on the growth in multicultural research and understanding, by noting its continued lack of overall representation in journals of psychology. Similarly, Atkinson, Morten, \& Sue (1993) claim that ethnic differences have been minimized and ignored in practice and research; and they recommend that articles on multicultural issues be represented in journals with more frequency and substance.

Ethical Considerations of Multicultural Counseling. The field of psychology has ethically bound itself to use culturally appropriate assessment and intervention to ensure unprejudiced treatment for clients from diverse backgrounds (American Psychological Association, 2003; Sue, Arrendo, \& McDavis, 1992). This ethical requirement is becoming ever more pressing considering “ethnic minorities” are estimated to become the numerical majority by 2050 (U.S. Bureau of the Census, 1995). These guidelines for working with diverse individuals are outlined by the ethical principles of psychologists and code of conduct of the American Psychological Association's (APA) (APA, 2003). The Competence standard (1.08), which directs psychologists serving members of, identified races and ethnicities to "obtain the training, experience, consultation, or supervision necessary to ensure the competence of their services” (p. 1601). The APA Guidelines for Providers of Psychological Services to Ethnic, Linguistic, and Culturally Diverse Populations (1993) further recommend that treatments be modified to more effectively treat and address issues particular to ethnic minorities. Counselors "need knowledge and skills for multicultural assessment and intervention” (p. 45), “to consider 
not only differential diagnostic issues but also the cultural beliefs and values of the client and his or her community in providing intervention” (p.46) and "to recognize ethnicity and culture as significant parameters in understanding psychological processes” (p. 47). These guidelines proceed to note that psychologists should be aware of their limitations in working with ethnic minority clients, increase their knowledge and awareness of multicultural issues, and seek consultation with, and/or make referrals to competent professionals when necessary.

Constructs of Multicultural Counseling. Even in light of these guidelines and standards, leaders in the field of counseling believe that the majority of White counselors are not competent at conducting adequate assessment, research, and interventions with ethnic minorities (Bernal \& Castor, 1994; Malgady, 1996). This is particularly due to counseling theory and counselor biases being bound almost exclusively to WhiteEurocentric perceptions and thought (Grieger \& Ponterotto, 1995; Helms, 1984). In an attempt to improve understanding of and service to persons of color, professionals attempted to define multicultural counseling competencies and standards. As part of a Division 17 task force, Sue, Bernier, Durran, Feinberg, Pedersen, Smith, and VazquezNutall (1982) wrote an influential paper calling for the development of multicultural competencies to handle these problems. Sue et al. (1982) addressed the shortcomings of multicultural counseling by outlining 11 multicultural competencies counselors need to posses in order to work with culturally diverse individuals. These 11 competencies have been well accepted in the field of counseling and are generally considered to be the minimum standard for training, research, and practice in counseling (Arredondo, Toporek, Brown, Jones, Locke, Sanchez, and Stadler, 1996). 
The 11 multicultural competencies were later refined into three basic constructs: awareness, knowledge, and skill (Sue, Arrendondo, \& McDavis, 1992). Awareness was identified as understanding one's core assumptions, principles, and prejudices based on one’s own cultural heritage as well as perceptions of other cultural systems. Knowledge was defined as comprehension of the worldview, values, and challenges of culturally dissimilar client. And, skill refers to the counselor's proficiencies of multicultural clinical skills and the use of appropriate intervention strategies and techniques based on the client's cultural and contextual factors. These three competencies have been broadly accepted in the field of counseling and used as guidelines for training, research, and practice (Sue, Carter, Casas, Foad, Ivery, Jensen, LaFromboise, Manese, Ponterotto, \& Vasquez-Nutall, 1998)

Although not adequately operationally defined (Atkinson, Morten, \& Sue, 1998), Arrendo, Toperek, Brown, Jones, Locke, Sanchez, and Stadler (1996) describe multicultural counseling as "the preparation and practices that integrate multicultural and culture-specific awareness, knowledge, and skills into counseling interactions.”(p. 43). Ponterotto, Casas, Suzuki, and Alexander (1995) used these three constructs to help them identify multicultural counseling competencies as being centered on “(a) understanding the different experiences of members of various cultural groups, (b) understanding the barriers to communication across cultures that exist as a result of these differences, (c) possessing a specific set of abilities that can potentially make a counselor culturally skilled” (p. 288). It is believed that if counselors have adequate understanding and can effectively employ all three of these constructs that they are considered to be multiculturally competent counselors (Holcomb-McCoy \& Meyers, 1999). 
Research on Multicultural Competence. The inquiry into multicultural competence has typically suggested two results: (a) ethnic minorities are more competent at multicultural counseling than Whites and (b) White counselors overestimate their competencies. Both of these observations suggest the importance of assessing and increasing multicultural competencies for White counselors in order to help them more effectively serve their ethnic minority clientele.

Comparison of Ethnic Minority and White Multicultural Competence. Research in the field of multicultural competence suggests that counselor ethnicity is a primary predictor of the level of counselor multicultural competence (Manese, 2001). HolcombMcCoy and Myers (1999) found that of five demographic variables (age, ethnicity, gender, educational level, and work setting) that the only counselor characteristic significantly related to multicultural competence was the counselor's ethnicity. Similarly, ethnicity was the only demographic variable Pope-Davis, Reynolds, Dings, and Nielson (1995) founded to be significantly related to multicultural counseling competencies. In particular, the body of literature in this area generally indicates that ethnic minorities are more competent than Whites at working with people of color. Ponterotto, Rieger, Barrett, and Sparks (1994) indicated that ethnic minorities score higher on multicultural competency measures than do Whites. Additionally, empirical evidence indicates that White counselors are not as multiculturally competent in their knowledge, awareness, and relationship as some ethnic minorities (Pope-Davis \& Ottavi, 1994). Sodowsky, Kuo-Jackson, Richardson, and Corey (1998), found very similar results in their study. The authors of the two studies found that Asian American and Hispanic graduate students in counseling scored significantly higher on multicultural 
knowledge in comparison to their White counterparts. The two studies also found that African American, Asian American, and Hispanic students scored significantly higher on multicultural awareness in comparison to their White counterparts. African Americans also scored significantly higher in multicultural relationships. However, the authors were unable to demonstrate that a difference existed based on ethnicity for multicultural skills.

As with the previous two studies, Pope-Davis, Reynolds, Dings, and Nielson (1995) found that ethnic minority interns scored higher in levels of awareness, knowledge, and relationships in comparison to the White interns. They also found that counseling psychology students, who receive more multicultural training, rated themselves as being more multiculturally competent than clinical psychology students. Pope et al. suggested from these findings that ethnic minority counselors and those who have received more multicultural training are likely to be more effective with ethnic minority clients. As with Pope-Davis and Ottavi (1994) and Sodowsky, et al. (1998), the authors failed to demonstrate that a difference existed based on ethnicity for multicultural skills.

Atkinson, Casas, and Abreu (1992); Gim, Atkinson, and Kim (1991); and Pomales, Claiborn, and LaFromboise (1986) also determined a significant effect between cultural sensitivity and perceptions of cultural competence. These studies reported that regardless of counselor ethnicity or participant acculturation, higher ratings in multicultural competence were given to counselors who were perceived to be culturally responsive, and lower rating were given to counselors who were perceived as culturally unresponsive. These findings suggest that counselors should be aware of cultural matters and respond to the cultural issues that ethnic minority clients present. 
Similarly, Okonji and Osokie (1996) reported that African American participants perceived African American counselors as being more effective and had more positive attributes than White American counselors. In addition, Sodowsky, Kuo-Jackson, and Richardson, and Corey (1998) determined that Hispanics had significantly higher scores on the Multicultural Counseling Inventory than did Caucasians and that the Caucasian had the lowest scores of multicultural competencies of the four ethnic groups studied.

White Counselor Multicultural Competence. White counselors overestimate their multicultural skills (Pope-Davis and Ottavi, 1994); and this overestimation tends to make White counselors susceptible to applying inappropriate theory and interventions in multicultural settings (Sue and Zane, 1987). Granello and Wheaton (1998) found that, with vocational rehabilitation counselors, that multicultural knowledge and awareness were significantly correlated with social desirability. The authors determined that due to social desirability, the White counselors tended to overestimate their actual multicultural competence. Further, the authors determined that the White counselors also overestimated their multicultural competence independent of social desirability. The authors concluded from this that the White counselors claimed knowledge and awareness of multicultural issues also may be elevated because they actually believe that they are more multiculturally competent than they actually are.

In addition to White counselors' tendency to over-estimate their multicultural competency, research on counselor multicultural competence also suggests that ethnic minorities are more competent at multicultural issues than Whites (Pope-Davis \& Ottavi, 1994). From these studies, it can be implied that not only are White counselors less multiculturally competent than ethnic minorities, but they actually are even less 
competent than they think. As a result, they are susceptible to applying interventions they believe to be culturally sensitive, which in actuality may be less appropriate and effective and potentially more damaging than they think.

General Versus Multicultural Competence. Experts in the field of multicultural competence have suggested that some confounding variables have had a strengthening effect on this body of literature while others have had a weakening effect (Constantine, 2002). Researchers investigating extraneous factors that impact multicultural competence identified co-variance between multicultural competence and counseling competence (Fuertes, 2001).

Determining if general counseling competency and multicultural competencies are distinct or identical constructs is one area of current debate. Fuertes (2001) stressed that a significant concern in the investigation and practice of multicultural competence is the need to distinguish them for general counseling competence. However, some investigators hold that there is evidence that suggests that general counseling competency and multicultural competency may be equivalent constructs. In Atkinson, Casas, and Abreu's (1992) study, the authors noted that the total scores on instruments that measure client's perceptions of counselor cultural competence and counselor general effectiveness had a .667 correlation with one another. From the high co-variability of general and multicultural counseling, the authors conclude that "The current findings could be construed as evidence that a counselor can ignore cultural content in a counseling session with an ethnic minority client and still be perceived by the client as credible source of help” (p. 518). Similarly, Coleman (1998) reported a strong correlation between the same general and multicultural competence. Coleman stated "that there is no distinction 
between general and multicultural counseling competence" and that "they may be a single construct” (p. 154). Coleman proceeded to suggest a need for more research in this area. Further, overlap of general and multicultural counselor competence can be inferred from Vinson and Neimeyer's (2000) findings that self-reported ratings of doctoral students of racial identity development were positively related with their ratings on general and multicultural counseling competencies.

Coleman, Wampold, and Casali (1995) suggest multicultural and traditional counseling competencies should be considered separate constructs. The authors state that "The essence of multicultural counseling is the ability to demonstrate to a client that their whole world, not just their psychological self, is understood and appreciated” (p.155). More recently, Constantine (2001) and Fuertes and Brobst (2002) found some evidence that suggests multicultural competence and general counseling competence have some common characteristics, but that they tend to be separate constructs. In her study Constantine determined that general and multicultural counseling share approximately $60 \%$ of their variance. Even with this overlap, she noted that these constructs are somewhat operationally distinct. Similarly, Fuertes and Brobst (2002), found a significant overlap for general and multicultural counseling competence in client satisfaction for ethnic minority and White clients. However, multicultural competence explained a significant amount of satisfaction variance for only the ethnic minority clients. This indicates that ethnic minority perceptions of counseling satisfaction have a stronger relation to multicultural counseling competence than that of Whites. The authors suppose that part of the difficulty in discerning multicultural competence from general competence is due to multicultural counseling competence existing within more 
subtle and sophisticated proficiencies that may be overshadowed by basic counseling skills and competence.

Inconsistencies and weakness in both the literature review on counselor multicultural competence indicates that further investigation needs to take place. Fuertes (2001) supports this assessment by noting the paucity of research that examines the relationship between multicultural competency with process and outcome measures of counseling. In agreement with the current literature review findings, Fuertes (2001) suggests that future research should include client's assessments of counselor multicultural competency "in relation to process and outcome indexes of counseling” (p.10). One area in particular that will be investigated in the current study is the relationship between multicultural competence of adventure based counselors and the corresponding counselor effectiveness as perceived by ethnic minority participant. The study also concurs with Fuertes recommendation of using field studies and quasiexperimental designs in order to access real-life situations and practical information for therapeutic outcomes with ethnically diverse individuals. Fuertes also encourages future research to investigate multicultural competence of counselors based on the client's experience. One method of achieving this is by examining the impact the counselor multicultural competence has on the clients' perceptions of the counselor's effectiveness. Counselor Effectiveness

In the recent past, there has been a surge of research investigating client's perception of their counselors level of competence as well as their level of satisfaction (Constantine, 2001). Over the past 25 years, various surveys have been devised to measure levels of counselor effectiveness (Ponterotto \& Furlong, 1985). These 
instruments, including one of the measures used in the present study, the Counselor Effectiveness Rating Scale, have typically been formulated on Strong's (1968) theory of counselor influence.

Constructs of Counselor Effectiveness. In his theory of counselor influence, Strong (1968) hypothesized that the outcome of therapy is directly related to the degree that clients view therapists as being an expert, attractive, and trustworthy. According to Strong: expertness is defined by the client's perception of the counselor's competence in his or her specialty; attractiveness is defined by the client's "perceived similarity to, compatibility with, and liking for" the counselor (p. 216); and trustworthiness is considered to be the client's perception of honesty, sincerity, openness, and lack of motivation for personal gain of the counselor. In general, the three main constructs of this theory have been empirically validated through dozens of studies (Gelso \& Fretz, 1992), and have been utilized as dependent variables in several investigations on counselor effectiveness ratings as perceived by their clients (Pope-Davis and Dings, 1995). One area of particular concern has been investigating counselor effectiveness and credibility with ethnic minorities.

Counselor Effectiveness with Ethnic Minorities. Investigation in this area is particularly important because the mental health needs of ethnic minorities have not been adequately met in application nor in research (Okonji \& Osokie, 1996). Inadequate treatment, access, scientific investigation to mental health needs for people of color is likely, in part, due to the general mistrust ethnic minorities have towards Whites (Watkins \& Terrell, 1988), traditional psychological theories and strategies being devised predominantly by White individuals for White individuals (Abreu, Gim, Chung, \& 
Atkinson, 2000), the paucity of ethnically diverse counselors in the profession (Tyler, Brome, \& Williams, 1991), and ethnic minorities receiving substandard care when they do seek out services (Ridley, 1989). To help substantiate some of these points from a historical perspective, Lewis Terman and Erick Erikson, two influential theorists were known to hold negative stereotypes of African Americans as well as the founding president of the APA, G. Stanley Hall, openly referred to "Black people as a 'primitive race in a state of immature development'” (Greene, 1986, p.48). Considering these to be conscious beliefs systems and motives, it is difficult to comprehend the extent that other forms of biases and discrimination have played in the development of psychology.

In order to increase utilization, decrease premature termination, and improve services it is important that ethnic minorities perceive the counseling setting to be safe and the counselor to be a credible source of help for their issues (Pedersen, 1988; PopeDavis \& Dings, 1991). In their study on the role of culture and cultural techniques in psychotherapy, Sue and Zane (1987) stress perceived counselor credibility as the most significant quality in working with ethnically diverse individuals. Examining ethnic minority perceptions of counselor effectiveness based on ethnic similarity-dissimilarity and multicultural competence are areas of research that have examined this issue.

Counselor Effectiveness Based on Counselor Ethnic Similarity/Dissimilarity.

Studies examining counselor ethnic similarity effect on client satisfaction and outcome of treatment is "equivocal" at best (Atkinson \& Masushita, 1991). According to Coleman, Wampold, and Casali (1995) the "particularist approach," clients preferring counselors from their own ethnic group, has not consistently been supported. Although the current 
review also found very mixed results in comparing the outcomes of the studies, the literature tended to lean in support of an ethnic similarity preference.

In an early review of counselor credibility with ethnic minorities, Atkinson (1983) noted that only two of the 11 studies found counselor credibility as being significantly related to counselor-client ethnic similarity. In addition, Atkinson’s (1985) subsequent review reinforced the inconsistent findings of studies in this area. Since Atkinson's $(1983,1985)$ reviews, several studies have professed counselor ethnic similarity and cultural sensitivity does impact clients' ratings of effectiveness and credibility. From their meta-analysis on ethnic minority ratings of ethnically similar and European American counselors, Coleman, Wampold, and Casali (1995) concluded that ethnic minority students tended to prefer ethnically similar counselors over European American counselors. However, Coleman et al. also reported that when asked to list the characteristics of the competent therapist, ethnic minority as well as White students place ethnic similarity below that of other characteristics; personality, attitudes, educational level, and maturity. The authors suggest that although ethnic similarity is a factor influencing preference, other characteristics tend to be more important. Atkinson and Lowe (1995) reached conclusions that were similar to those of Coleman et al. in their review of literature. They evaluated existing literature and reported that with other factor being equal, ethnic minority clients prefer ethnically similar therapists and that ethnic minorities don't solely base their preference and satisfaction of therapist based solely on their ethnicity.

A number of studies also indicated that ethnically similar counselors were rated as more effective and credible than ethnically dissimilar counselors. A group of studies 
investigating client/counselor ethnic similarity found that African Americans rate ethnically similar counselors as more expert, trustworthy, and attractive (Atkinson, 1983 ; Atkinson \& Lowe, 1995; Okonji \& Osokie, 1996). In examining high dropout rates of Black clients with White counselors, Watkins and Terrell (1988) determined mistrust level significantly interacted with counselor race. Specifically they found that highly mistrustful Blacks expected White counselors to be less accepting, trustworthy, and expert; and they expected less in terms of counseling outcomes than when the assigned counselor was Black. Similarly, Watkins, Terrell, Miller, and Terrell (1989) found that African Americans low as well as high on mistrust viewed White counselors as being less credible and less likely to be able to help them with their problems.

In their comprehensive review of studies on ethnic preferences of Mexican American clients, Lopez, Lopez, and Fong (1991) found that the Mexican American clients preferred ethnically similar counselors. They claimed that Mexican American clients perceived ethnically similar counselors as more competent than ethnically dissimilar counselors. In a study using Mexican Americans college students, Ponce and Atkinson (1989) found that the participants rated ethnically similar counselors higher than White Counselors, and noted that they were more willing to see an ethnically similar counselor for personal, academic, and vocational concerns. Further, Ruelas, Atkinson, and Ramos-Sanchez (1998) found that for Mexican Americans, ethnic background and adherence to cultural norms and behaviors are related to perceptions of counselor effectiveness. In particular, they found that Mexican Americans rated counselors with similar ethnicities and adherence to similar cultural norms and behaviors to be more 
credible, expert, and trustworthy. The authors also determined that acculturation to North American culture did not significantly affect ratings of counselor credibility.

However, in contrast to the previous findings, a number of studies have failed to demonstrate a positive relationship between counselor ethnic similarity and cultural sensitivity with clients’ ratings of effectiveness and credibility. Atkinson, Poston, Furlong, and Mercado (1989) conducted a study, using 339 students from various ethnicities that investigated ethnic group preferences for various counselor characteristics. In a list of 16 counselor characteristics, counselor ethnic similarity ranked fifth in level of importance, coming behind education level, similar attributes, age, and similar personality. The authors concluded from this that ethnicity plays a very small role in the preferences individuals have for counselor characteristics. Further, Atkinson, Ponce and Martinez (1984) and Atkinson, Winzelberg, and Holland (1985) failed to find significant support that Mexican American individuals view ethnically similar counselors as being more credible than ethnically dissimilar counselors. Hess and Street (1991) also failed to find any significant variance in Mexican American high school students' ratings of ethnically similar and dissimilar counselors. Atkinson, Casas, and Abreu’s (1992) results used Mexican American participants to determine the relationship between acculturation, counselor ethnicity, and counselor cultural sensitivity, with perceived counselor competence. The authors failed to find a differences for ratings based on counselor ethnicity for all experimental conditions. Ramos-Sanchez, Atkinson, and Fraga (1999) also failed to demonstrate a significant difference in Mexican American college students’ perceptions of ethnically similar or dissimilar counselor credibility. They also failed to demonstrate a difference in perceived credibility between counselors 
who spoke English and those who spoke Spanish. However, the ratings of both counselor credibility and cross-cultural competence were found to be a function of the participant's bilingual ability. Their findings also suggested that, as Mexican American acculturate, their perceptions of counselor credibility and cross-cultural competence diminished.

It is likely that the results from this body of research are mixed because a number of confounding factors (e. g. , acculturation and racial identity) may be affecting the perceptions of these potential clients (Pope-Davis, Liu, Toporek, \& Brittan-Powell, 2001). Although the literature on counselor ethnic preference tends to indicate an effect of ethnic similarity, inconsistent results seem to suggest that the body of research remains inconclusive and additional research is warranted.

Counselor Effectiveness Based on Counselor Multicultural Competence. Little information exists addressing ethnic minority client's attitudes towards and satisfaction with counseling based on multicultural competence (Constantine, 2002; Fuertes, 2001); and the studies that do exist in this body of research have also tended to demonstrate inconsistent results to one another. As this body of literature grows, the more recent studies have tended to find more consistent support for a positive relationship with multicultural competence and ethnic minority counseling satisfaction.

Gim, Atkinson, and Kim (1991) determined that Asian American college students rated culturally sensitive counselors as being more credible (i.e. expertness, trustworthiness, and attractiveness). The authors failed to find an effect for levels of acculturation and counselor ratings. While investigating counselor rating based on levels of multicultural competence, Pomales, Claiborn, and LaFromboise (1986) found empirical support that African American college students rated culturally sensitive 
counselors as being more culturally competent as well as having higher expertness, but not trustworthiness or attractiveness, than culture-blind counselors, regardless of the student's racial identity development. In the attempts to replicate these studies, Atkinson, Casas, and Abreu’s (1992) results used Mexican American participants to determine the relationship between acculturation, counselor ethnicity, and counselor cultural sensitivity, with perceived counselor competence. The authors determined a significant cultural sensitivity effect for perceptions of cultural competence. Ethnically diverse participants positively correlated ratings of counselor competence with cultural responsive therapists, but no differences were found for ratings based on counselor ethnicity.

In a similar study, Sodowsky (1991) determined that Asian and White students, who observed videotaped counseling sessions, perceived counselors who displayed culturally similar values as more expert and trustworthy. However, the author found no differences in ratings for Korean students who observed both conditions. Of note, Sodowsky also found that White students prefer the Western counseling perspectives.

Recent studies have found more consistent support that counselors who are perceived as multiculturally competent are generally considered to be more effective by ethnic minorities. In a study by Coleman (1998), an ethnically diverse group of college students rated counselors who were culturally sensitive as being more effective than counselors who were culturally neutral in both general and multicultural counseling competency. Coleman also indicated that ethnic minorities perceived culturally neutral counselors as being less competent. Consistent with Coleman’s findings, Fuertes and Brobst (2002) also found that perceptions of counselor multicultural competence correlated strongly, nearly $50 \%$ of the variance, with the counselor's level of 
attractiveness, expertness, and trustworthiness. Zhang and Dixon (2001) found that Asian students, who received culturally responsive interventions, rated their counselors as significantly more expert, attractive, and trustworthy than culturally neutral interventions. With these inconsistencies, methodological limitations, and Coleman et al.'s (1995) warning not to take the results of minority perceptions of counselor credibility and effectiveness at "face value"; further research in this area needs to be conducted in a resolute manner. Naturally occurring phenomena, the complexity of multicultural counseling, and methodological issues have been suggested as being responsible for the inconsistent findings in this field of study (Abramowitz \& Murray, 1983; Atkinson, 1985). For instance, multicultural clients' within-group differences seem to be partially responsible for conflicting results in the literature (Lopez, Lopez, and Fong, 1991). PopeDavis and Dings (1995) tentatively conclude for some individuals within each of the ethnic minority groups, counselor similarity/dissimilarity is an important factor in determining perceived counselor credibility while it is not for others. This suggests that the differences in perceived counselor effectiveness by ethnic minorities is significantly influenced by the differences that exist between the members of particular ethnic groups. Another possible cause for the inconsistent results is related to methodology of studies in this field. Coleman, Wampold, and Casali (1995) state, in their meta-analysis of perceptions of ethnically similar and dissimilar counselors, that "method was clearly related to effect size" (p. 60). Coleman, et al. conclude: "this heterogeneity and sensitivity to method suggests that we have not yet isolated a clear and consistent pattern of results that can be taken at face value” (p. 60). 
Studies investigating this area have also tended to use measures that evaluate client's perceptions of counselor competence indirectly and by inference (Fuertes, 2001). Fuertes suggests that many of these problematic issues could be resolved by directly assessing client's perceptions of their counselor's multicultural competence. Fuertes proceeds to stress the importance of future research to include "the client's voice... to acknowledge, respect, and incorporate the client's experience into the process of helping” (p. 12). The client's perceptions of counselors' level of effectiveness are essential in order to improve the process and outcome of therapy for ethnically diverse individuals. One such instrument that is being used in the current study, which examines client perceptions of counselor effectiveness is the Counselor Effectiveness Rating Scale.

Literature on counselor effectiveness with ethnically diverse populations has examined counselor ethnic similarity/dissimilarity effect as well as multicultural competence effect. The collection of literature in this area has tended to be mixed at best. Consistent with past reviews of the literature on the relationship with effectiveness ratings and counselor ethnicity and cultural competence, the current review has found inconsistent results. These inconsistencies and a lack of research in this area regarding ethnic minority youth at risk and their level of satisfaction based on their counselors multicultural competence warrants research. 


\section{CHAPTER THREE}

Method

\section{Introduction}

This study was designed to investigate the relationship between adventure based counselors' multicultural competencies and effectiveness ratings by participants, particularly ethnic minority participants. The study was conducted at a program that facilitates 30-day adventure based courses for youth at risk. Data collection for this investigation was completed from June 2002 to September 2003.

This chapter included selection criteria and methods of accessing the participants and counselors recruited for the study. The Instruments that operationally defined variables of the study are described in the dependent and independent variables sections. The Procedure section delineated how the instruments were administered as well as the day-to-day events and proceedings of the adventure based program. The Research Design section identified how the relationship between the dependent and independent variables were analyzed. The Statistical Analysis section outlined the methods of statistical analysis. A table of Independent and Dependent Variables visually displayed the relationship between the variables and the statistical analyses employed.

\section{Selection of the Youth at Risk Participants}

The youth at risk participants were accessed from an adventure based program that has been in operation for over 25 years in a Midwestern state. This program is described in Appendix A. Theoretical underpinnings of the program are described in Appendix B. 
Participants typically were referred by probation officers working for the United Delinquent Intervention Services, by caseworkers of the Department of Child and Family Services, and by private social service agencies. The participants were predominantly either adjudicated and/or from abusive or neglectful homes. All of the participants who successfully completed the 30-day program were invited to participate in the study.

The participants were typically referred because of some behavioral, legal, familial, or substance abuse problem. Prior to participant inclusion, the counselors conducted an intake interview at the referring agent's office in the participant's hometown. A substantial history was gathered during the interview, and the adolescent was assessed for appropriateness for the program. According to the program's advertising pamphlet, "Youth must have no severe physical or mental disability that might endanger the youth or prevent successful completion of the program” (Spectrum Wilderness Programs, 2004). After viewing an informative slide show on the components of the course, the prospective participant was informed that program involvement was voluntary and that he or she had to sign a behavior contract to be considered for the program. However, the term "voluntary" is debatable, considering that it is common for the referring agent to offer participation in the course as an alternative to a more aversive consequence (e.g., incarceration, not being able to return home, being taken out of his or her home).

\section{Selection of the Counselors}

The participating counselors were accessed from the staff of the adventure based

program. All counselors leading courses during the data collection period were invited to participate in the study. No criteria was set for excluding any counselors from the study. 
Due to the number of courses run over the consecutive months, and the limited number of staff, there were a number of counselors who lead more than one of the groups included in the study. The adventure based program used three counselors per course, who worked directly with the participants for 30 straight days.

The full-time staff typically participated in approximately 20 days of winter and summer training, and the seasonal staff participated in approximately 10 days of staff training prior to working with the participants. The staff trainings typically were comprised of therapeutic, wilderness, and safety skills training. According to the program’s coordinator, the staff trainings did not include any formal multicultural components.

\section{Treatment Setting}

The adventure based program, Spectrum Wilderness Program (SW) at Touch of Nature Environmental Center (TON) - Southern Illinois University (SIU) was created in 1969 with consultation from Outward Bound (OB) (Spectrum Wilderness Programs, 2004; Wichmann, 1991). Thus, much of the wilderness program’s philosophy is based on the OB model and has to abide by SIU's guidelines. Wichmann (1991), one of the founders and directors of SW, outlines the fundamental components of SW (see Appendix B). The SW staff consist of a program coordinator, assistant program coordinator, a course director, eight full-time staff, and 16 seasonal staff. The program typically runs eleven 30-day courses a year with three counselors and one logistician per course and vary between six to 11 participants per course. 


\section{Measurement of Dependent Variable}

The dependent variable is the participants' perceived effectiveness of their adventure based counselors. This variable is further divided into four subcategories: Expertness, Attractiveness, Trustworthiness, and Total Effectiveness (Strong, 1968). These dependent variables are operationalized by the Counselor Effectiveness Rating Scale (Atkinson \& Carskaddon, 1975).

Counselor Effectiveness Rating Scale (CERS). The CERS is a 10-item semantic differential questionnaire that rates counselors on Expertness, Attractiveness, Trustworthiness, and Total Effectiveness (Atkinson \& Carskaddon, 1975). The instrument takes less than five minutes to complete. The CERS is based on the counselor influence theory of Strong (1968) and the communication theory of Hovland, Janis, and Kelley (1953). The three sub-scales consist of three items each, with the remaining item designed to measure willingness to see a counselor. Each concept is rated on a 7-point bipolar scale from (1) an unfavorable rating to (7) a very favorable rating. The score of each sub-scale is determined by computing an average of the three corresponding items.

The internal consistency was reported with a Cronbach's alpha coefficient of .88 for the Expertness, .78 for Attractiveness, .75 for Trustworthiness, and .90 for the Total CERS score (Atkinson \& Wampold, 1982). Atkinson and Wampold further reported a concurrent validity of .80 by correlating the scores on the Counselor Rating Form with the scores of the CERS. It should be noted that Ponterotto and Furlong (1985) caution about using the three dimensions as independent measures due to their high intercorrelations. 
With permission from the author, Donald Atkinson, the CERS was modified to be more specific to the setting, the adventure based counselors, and the participants completing the questionnaire. The wording was changed to simplify the definitions, such that the meaning of the factors remained. According to the Atkinson, through personal correspondence, such changes did not affect the validity or reliability of the instrument (Atkinson, 2001).

\section{Measurement of Independent Variables}

The three independent variables for the study are the demographic variables of the youth at risk and counselor participants as well as the counselors’ self-reported multicultural competencies. One independent variable, participant ethnicity, was categorized into either (a) White or (b) Ethnic Minority (i.e., African American/Black, Asian American, Hispanic/Latino/Latina, Native American, and Bi-racial). Ethnic category was determined from the Wilderness Participant Research Questionnaire. This instrument can be found in Appendix C. The script that was read to Participants when the Wilderness Participant Research Questionnaire was administered can be found in Appendix D. The second independent variable, counselor demographics, was collected with the Wilderness Counselor Research Questionnaire. The Multicultural Counseling Knowledge and Awareness Scale assessed the third independent variable, counselor multicultural competence.

Wilderness Participant Research Questionnaire. Besides identifying ethnicity, the demographic questionnaire for the participants (see Appendix C) also was used to identify the participants' age and gender. A script was read during the administration by a proctor (see Appendix D). The proctor was typically the course logistician and not a 
counselor for the particular course. The investigator developed the Wilderness Participant Research Questionnaire.

Wilderness Counselor Research Questionnaire. Besides identifying ethnicity, the demographic questionnaire for the counselors (see Appendix E) also was used to identify the counselors’ age, gender, education, and work experience.

Multicultural Counseling Knowledge and Awareness Scale. The Multicultural Counseling Knowledge and Awareness Scale (MCKAS) (Ponterotto, Gretchen, Utsey, Rieger, \& Austin 2000) is a 32 item questionnaire developed to measure multicultural competence dimensions based on the multicultural competencies specified by Sue et al. (1992). The MCKAS takes about 20 minutes to administer and was completed by the counselors prior to the courses they facilitated.

The items of the MCKAS are worded in general terms regarding multicultural issues rather than toward specific ethnic groups. Its scoring system records subject responses to their perceived multicultural competence on a 7-point continuum scale that ranges from (1) not at all true to (7) totally true. The factors consist of two sub-scales, Multicultural Awareness and Knowledge/Skill, with higher scores indicating higher multicultural competency with the corresponding sub-scales. The Awareness sub-scale consists of 12 questions with a total scoring range from 12 to 84 . The Knowledge/Skill sub-scale consists of 20 questions with a total scoring range from 20 to 140 . No classification of scores to determine various levels of multicultural competence was found in MCKAS literature.

The MCKAS was created due to psychometric concerns with its predecessor, the MCAS-B. Ponterotto et al. (2000) performed a large-sample confirmatory factor analysis 
of the MCAS-B. The investigators attempted to broaden its applicability by accessing 525 participants that represented diverse demographic categories; including various ages, ethnicities, genders, educational levels, and multicultural training level. The results indicated a two-factor oblique model. This consisted of the two subscales: awareness and knowledge. The goodness of fit index $=.75$ and coefficient alphas was .79 for awareness and .92 for knowledge. Inter-correlation between the subscales was found to be low ( $\mathrm{r}=$ .20) and supported the orthogonal model of factor extraction.

Validity for the MCKAS was found to be at moderate to high levels of criterion related validity, which was determined by examining within group comparisons of multicultural experts to practicing professionals and students. Experts in the field had higher scores than practitioners and students.

\section{Research Design}

Each youth participant (Ethnic Minority or White) rated three counselors (varying in Multicultural Competence) on four dimensions of Counselor Effectiveness: attractiveness, expertness, trustworthiness, and total effectiveness. The counselors completed surveys that rated themselves on three dimensions of Multicultural Competence: knowledge, awareness, and total multicultural competence. Due to the parametric assumptions, of normal distribution, not having been met, the primary research design is a correlational analysis utilizing Kendall’s Tau.

\section{Statistical Analysis}

A Kendall's Tau was used as the primary statistical procedure due to in being a nonparametric analysis. The independent variables consisted of Participant Ethnicity (Ethnic Minority versus White), counselor demographics, and Counselor Multicultural 
Competency (Knowledge Score, Awareness Score, and Total). The dependent variable consisted of Counselor Effectiveness (Attractiveness Ratings, Trustworthiness Ratings, Expertness Ratings, and the Total).

Procedure

Approval for the research was secured from the Program Coordinator of the wilderness program, Southern Illinois University’s Institutional Review Board, and West Virginia's Institutional Review Board. Due to the use of adolescent participants consent and assent forms were created for inclusion in the study. The board conducted a quorum review for approval. The investigator received a list of the courses scheduled for the 2002 and 2003 calendar. Prior to the courses the investigator sent questionnaire packets to the Program Coordinator with each, including three Wilderness Counselor Research Questionnaires (see Appendix E) and 3 MCKAS surveys, one for each counselor assigned to each course. This was repeated for each of the 12 courses included in the study.

Prior to the course, during one of the three days of course preparation, the Program Coordinator distributed the research instruments to the course counselors during an activity, called "staff talks." At that time the course counselors completed the research instruments, and enclosed them in a tamper-proof envelope, addressed to the investigator. Based on statistical concerns and practicality, the counselors would only complete the MCKAS once, even though some of them would participate in more than one course included in the study.

Prior to the courses, the investigator prepared participant packets that include a Participant Research Questionnaire and 3 CERS instruments for every youth at risk 
participant to rate each of the counselors individually. Each mailing included 12 packets per course. The logistician (see Appendix A) for each course was given the participants’ packets and distributed them during the day prior to each participant's graduation from the course. At the time of distribution he or she read the Wilderness Questionnaire Script and Administration Directions (Appendix D). Once participant instruments were completed, they were enclosed in a tamper-proof envelope and mailed to the investigator. 


\section{CHAPTER FOUR}

Results and Discussion

Results

The present investigation was designed to examine the relationship between adventure based counselor multicultural competence and their level of effectiveness as rated by ethnic at risk minority youth. This chapter will present the results of this investigation, and how its analysis was modified to fit statistical limitations of the data gathered from the research participants.

The results of the analysis are presented in four separate sections in this chapter. The first section presents information on data collection and demographic data of the samples. The second section examines characteristics of the data from the Counselor Effectiveness Rating Scale (CERS) through descriptive and nonparametric analysis. The third section explores the characteristics of the data from the Multicultural Counseling Knowledge and Awareness Scale (MCKAS) through descriptive and inferential analysis. The fourth section explores features of the CERS together with the MCKAS through descriptive and nonparametric analysis.

\section{Data Collection and Demographic Data}

The data were gathered from 12 thirty-day adventure based programs that took place between June, 2002 and July, 2003. During this period of time, 14 courses were completed in which data could have been gathered. Data were not collected on 48 participants. Eleven of these youths were removed from the courses, and the remaining 37 were not included due to logistics associated with outdoor programs, including the need to evacuate participants because of a forest fire. The reasons that the 11 youths were 
removed was not documented, but it is likely that most of them were removed due to severe behavioral problems. This resulted in obtaining data on 86 youth from a potential pool of 134 .

The youths included in the study ranged in age from 13 to 18 years old with an average age of 14.9 years old. They consisted of 78.7 percent male $(n=68)$ and 21.3\% female $(n=18)$. The youth were 60.1 percent White $(n=52)$ with 39.9 percent ethnic minority $(n=34)$. Of the ethnic minorities, 46.2 percent was African American ( $n=16)$, 35.2 percent was Latino/Latina $(\mathrm{n}=12), 23.1$ percent was biracial $(\mathrm{n}=8)$, and 6.6 percent was Native American ( $\mathrm{n}=2)$.

Of the potential counselor subject pool, consisting of 27 counselors, 5 did not participate, resulting in a sample of 22 counselors. Although some of the counselors led more than one course included in the study, they were only administered the MCKAS once. As a result, 13 of the data sets included were based on previous MCKAS response sets. The resulting 35 data sets were included in the present study. The counselors' ages ranged from 21 to 36 years old with an average age of 24.8 years of age. The gender makeup of the counselors was 45.7 percent female $(n=16)$ and 54.3 percent male $(n=$ 19). The ethnic makeup of the counselors consisted of 8.6 percent ethnic minority $(\mathrm{n}=$ 3) and 91.4 percent White $(n=32)$. The highest degree acquired by the counselors consisted of 17.1 percent with high school diploma $(n=6), 5.7$ percent with associate's degree $(n=2), 74.3$ percent with bachelor's degree $(n=26)$, and 2.9 percent with a master's degree $(n=1)$. Of those with degrees, 40.0 percent were in outdoor recreation $(n=14), 11.4$ percent were in social sciences $(n=4), 14.3$ percent were in public relations $(n=5), 17.1$ percent were in the sciences $(n=6)$, and 3.1 percent were in 
business $(n=1)$. The counselor experience level, leading outdoor courses, consisted of a range of 30 to 505 days with an average employment with Touch of Nature being 221

days. The number of 30 day courses competed by counselors averaged 3.94. The number of days working with youth at risk in a wilderness setting ranged from zero to 270 with an average of 98 days.

There is a noticeable discrepancy with the ratio of ethnic youths to ethnic minority counselors as well as with the ratio of courses that have ethnic minority counselors to those with only White counselors. While the participants consisted of 39.9 percent ethnic minority ( $n=34)$, only 8.6 percent of the counselors were ethnic minorities $(n=3)$. This is an under-representation of 4.6 ethnic minority youths to each ethnic minority counselor. Further, only one in four $(n=3)$ of the 12 courses included in the study had ethnic minority counselors.

\section{Counselor Effectiveness Rating Scale}

After examining the distribution of scores for the dependent measure, it was determined that the range of scores was exceedingly restricted and heavily skewed to the left (i.e. most scores fell on the "Good" end of the scale). After computing the ranges, medians, means, and standard deviations for Expertness, Trustworthiness, Attractiveness, and Total Effectiveness for the CERS, it was concluded that the narrow distribution of the scores could adversely impact inferential analyses of the data. These statistical problems could increase the likelihood of Type 2 error, result in misleading conclusions, and/or produce results with scarce applicability and practicality. 
The range of data for the categories of the CERS are shown in Table 1. The sum of the Total CERS score was the combined scores of Expertness, Attractiveness, Trustworthiness, and an item on the questionnaire that measured desire to continue the relationship.

Table 1

Counselor Effectiveness Ratings by At Risk Youth Participants $(n=2530 *)$

\begin{tabular}{lcccccc}
\hline & & & & & & \\
& Minimum & Maximum & Median & Mean & $\begin{array}{c}\text { Standividual } \\
\text { Deviation }\end{array}$ & $\begin{array}{c}\text { Score } \\
\text { Mean }\end{array}$ \\
\hline Expertness & 12.0 & 21.0 & 20.0 & 19.1 & 2.0 & 6.4 \\
Attractiveness & 8.0 & 21.0 & 19.0 & 18.5 & 2.7 & 6.2 \\
Trustworthiness & 9.0 & 21.0 & 19.0 & 18.8 & 2.3 & 6.3 \\
Total & 38.0 & 70.0 & 64.0 & 62.6 & 6.9 & 6.3 \\
\hline
\end{tabular}

*Note: $\mathrm{n}$ is the number of individual youth at risk CERS responses

Inspecting the individual responses of youth ratings further helps to illustrate the degree of the data's skewness. Out of the 2530 individual responses, based on a seven point Likert scale, only 2 percent of the scores fell in the bottom 3 indices, while 98 percent fell in the remaining top 4 indices (see Table 2). 
Table 2

At Risk Youth Participant Rating of Counselor Effectiveness by Numeric Scale Items

\begin{tabular}{ccc} 
Ratings & Responses & Percent \\
1 & 2 & 0.1 \\
2 & 9 & 0.4 \\
3 & 23 & 0.9 \\
4 & 107 & 4.4 \\
5 & 360 & 15.3 \\
6 & 681 & 29.0 \\
7 & 1,348 & 54.7 \\
Total & 2,530 & 100.0 \\
\hline
\end{tabular}

Additionally, the restricted nature of the scores constricted the variance of the dependent variable from a seven-point Likert scale to one that only represents the top four indices of the instrument. In a normal distribution, the median of a seven-point scale should be four; however, in the current data is the median has a value of six. Consequently, the scale shifted from its original range of seven points indicating from "bad” to "good" to one of less than four points, merely differentiating between "above average” and “good.” As a result, determining any legitimate differences between the 
levels of counselor effectiveness in regard to multicultural competence became highly unlikely, and the potential for finding misleading results beyond an acceptable level. Further, if any legitimate differences were concluded through analyzing the current data, it is likely that it would be neither useful nor practical to differentiate counselors in regards to those rated "good" from those rated "really good."

According to Kerr, Hall, \& Kozub (2002), if z-value for skewness, dividing the skew value by its standard errors, is better than \pm 1.96 , then the data significantly deviates from a normal distribution. The computed CERS z-values for skewness in regards to expertness was -6.0 , attractiveness was -8.8 , trustworthiness was -8.0 , and total effectiveness was -6.4 (see Table 3). These scores indicate that the data significantly deviate from a normal distribution and probably are not representative of the population. Consequently, parametric analysis should not be computed due to the assumption of equal variance not being met. Using a MANOVA to analyze the current data, as originally planned, is contraindicated because it would violate one of the necessary parametric assumptions. 
Table 3

Skew Values for Total Counselor Effectiveness Ratings by At Risk Youth Participants

Skewness Standard Error for Skewness

\begin{tabular}{lcc} 
Expertness & -0.9 & 0.2 \\
Attractiveness & -1.4 & 0.2 \\
Trustworthiness & -1.2 & 0.2 \\
Total & -1.0 & 0.2 \\
& & \\
\hline
\end{tabular}

Due to the skewness, restricted range, and inflated response of scores for the CERS related data, the investigator used the median for descriptive analysis. For skewed data, the median rather than the mean is typically a more accurate measure of location, and with restricted ranges of data the median is less deceptively influenced by outliers than the mean (Utts \& Heckard, 2004). With the current data set, a score typically considered to be "average" (i.e. a score of four) would be considered an outlying value due to the clear restriction of scores and negative skewness of the data set. This results in a high potential for typically "average” ratings to pull down the mean and mislead any subsequent analysis. This is supported by the discrepancy between the Total CERS mean being 62.6 and its median being 64.0 . 
Probably the most striking feature of the current data is the median score of 64 out of 70 possible points. This indicates that the participants rated the counselors as being exceptionally effective in all areas that the CERS measured. The possibility of confounding variables is addressed in the discussion section.

Kendall's Tau was used as a non-parametric means of analysis due to the restricted range of the CERS. No significant relationships were determined between youth at risk ethnicity and levels of the various categories of counselor effectiveness (see Table 4).

Table 4 Counselor Effectiveness Means/Medians by Youth at Risk Ethnicity $(n=86)$

Expertness Attractiveness Trustworthiness Total

\begin{tabular}{lllll}
\hline Ethnic Minorities $(\mathrm{n}=34)$ & $18.8 / 20.0$ & $18.7 / 19.0$ & $18.7 / 19.0$ & $62.2 / 64.0$ \\
Whites $(\mathrm{n}=52)$ & $19.4 / 20.0$ & $18.4 / 19.0$ & $18.9 / 19.0$ & $62.9 / 64.0$
\end{tabular}

Examining counselor CERS ratings based on the age of the participants suggests that that trustworthiness was the only factor related to the age of the participant $(r=-0.14, \mathrm{p}<.05)$ The findings suggest that perceived counselor trustworthiness decreases as the youth at risk got older (see Table 5). 
Table 5

Counselor CERS Mean/Median Ratings by Age of Youth at Risk Participants ( $\mathrm{n}=86)$

\begin{tabular}{lcccc}
\hline & & & & \\
Age & Expertness & Attractiveness & Trustworthiness & Total \\
\hline & & & & \\
13 & $19.5 / 20.0$ & $19.1 / 20.0$ & $19.3 / 20.0$ & $64.0 / 67.0$ \\
14 & $19.1 / 20.0$ & $18.5 / 19.0$ & $18.9 / 20.0$ & $62.7 / 65.0$ \\
16 & $19.2 / 20.0$ & $18.7 / 19.0$ & $19.0 / 20.0$ & $63.0 / 64.0$ \\
17 & $19.1 / 20.0$ & $18.4 / 19.0$ & $18.5 / 19.0$ & $62.1 / 64.0$ \\
18 & $18.9 / 19.0$ & $17.2 / 18.0$ & $18.0 / 18.0$ & $60.2 / 60.5$ \\
\hline
\end{tabular}

Although the scores given by the female participants were not significantly different from the male participants, the male participants rated the counselors slightly higher in all categories of counselor effectiveness than did the female participants (see Table 6). 
Table 6

Counselor Effectiveness Mean/Median Ratings by Gender of Youth at Risk $\quad(\mathrm{n}=86)$

Gender Expertness Attractiveness Trustworthiness Total

$\begin{array}{lllll}\text { Females }(\mathrm{n}=10) & 18.9 / 20.0 & 18.3 / 19.0 & 18.5 / 19.0 & 61.7 / 63.0 \\ \text { Males } \quad(\mathrm{n}=76) & 19.2 / 20.0 & 18.5 / 19.0 & 18.9 / 20.0 & 62.8 / 65.0\end{array}$

Counselor demographics did not appear to have a significant impact on their perceived level of effectiveness as determined by youth at risk participants (See Table 7).

Table 7

Correlations between Counselor Demographics and Counselor Effectiveness $(\mathrm{n}=35)$

Demographics Expertness Attractiveness Trustworthiness CERS Total

\begin{tabular}{lllll} 
Age & 0.19 & 0.06 & 0.19 & 0.18 \\
Gender & -0.26 & -0.02 & 0.18 & -0.16 \\
Race & 0.09 & 0.10 & 0.08 & 0.10 \\
Degree & -0.15 & -0.14 & -0.19 & -0.16 \\
Discipline & 0.04 & -0.14 & 0.02 & -0.03 \\
Employment & 0.04 & 0.04 & 0.04 & 0.02 \\
* Indicates Significance at .05 level (Kendall's Tau Correlation, 2 tailed) \\
\hline
\end{tabular}


Multicultural Counseling Knowledge and Awareness Scale

Visual inspection of the MCKAS scores suggested a relatively normal distribution of values. Computation of all z-values for skewness for MCKAS resulted in acceptable levels for parametric analysis, so inferential analysis could be conducted in good faith (see Table 8). Descriptive and inferential analyses were conducted for various counselor variables and their corresponding MCKAS scores.

Table 8

Skew Values for Counselor Multicultural Competence Results

Constructs Skewness Standard Error for Skewness

$\begin{array}{lll}\text { Knowledge } & -0.34 & 0.31\end{array}$

$\begin{array}{lll}\text { Awareness } & -0.15 & 0.31\end{array}$

$\begin{array}{lll}\text { Total } & -0.57 & 0.31\end{array}$


The counselors appeared to score higher on levels of knowledge in comparison to their levels of awareness (see Table 9).

\section{Table 9}

Counselor Multicultural Knowledge and Awareness Scores

$(n=35)$

\begin{tabular}{|c|c|c|c|c|c|c|}
\hline Construct & Minimum & Maximum & Median & $\begin{array}{c}\text { Individual } \\
\text { Score } \\
\text { Mean } \\
\end{array}$ & $\begin{array}{l}\text { Overall } \\
\text { Mean }\end{array}$ & $\begin{array}{l}\text { Standard } \\
\text { Deviation }\end{array}$ \\
\hline Knowledge & 50.0 & 121.0 & 88.0 & 4.4 & 88.7 & 20.1 \\
\hline Awareness & 24.0 & 57.0 & 42.0 & 3.6 & 43.5 & 9.2 \\
\hline Total & 84.0 & 166.0 & 144.0 & 4.1 & 132.2 & 23.9 \\
\hline
\end{tabular}

Significant relationships were determined for counselor degree and multicultural knowledge $(r=-0.29, \mathrm{p}<.05)$, and for multicultural awareness length of employment $(r=$ $-0.42, \mathrm{p}<.05)$ and number of course led $(r=-0.28, \mathrm{p}<.05)$ (see Table 10$)$. 
Table 10

Correlations between Counselor Demographics and Multicultural Competence $(\mathrm{n}=35)$

\begin{tabular}{lccc} 
Demographics & Knowledge & Awareness & $\begin{array}{c}\text { Total Multicultural } \\
\text { Competence }\end{array}$ \\
\hline Age & 0.18 & -0.16 & 0.01 \\
Gender & -0.22 & 0.07 & -0.11 \\
Race & 0.06 & -0.15 & -0.06 \\
Degree & $-0.29 *$ & -0.10 & 0.25 \\
Employment & -0.05 & $0.42^{*}$ & 0.05 \\
Courses & -0.04 & $0.28^{*}$ & 0.01 \\
\hline
\end{tabular}

*Indicates Significance at .05 level (Kendall’s Tau Correlation, 2-tailed)

Counselor Effectiveness and Multicultural Competence Analysis

Nonparametric correlations were computed using rank order analysis with Kendall's Tau because parametric assumptions were not met for the CERS. The results indicate a number of significant negative relationships. The significant negative relationships included a) Expertness with Awareness and Total Multicultural Competence; b) Attractiveness with Knowledge and Total Multicultural Competence; c) Trustworthiness with Knowledge, Awareness and Total Multicultural Competence; and d) Total Counselor Effectiveness with Knowledge, Awareness and Total Multicultural Competence. The results indicate that as most indices of counselor multicultural competence increases their levels of effectiveness with the youth at risk participants 
decreases. However, the relationships were generally weak, with the strongest correlation only explaining $2.8 \%$ of the shared variance (see Table 11 ).

Table 11

Correlations of CERS and MCKAS Scores for all Youth at Risk ( $\quad(\mathrm{n}=86)$ MCKAS

\begin{tabular}{llll}
\multicolumn{1}{c}{ CERS } & Knowledge & Awareness & Total \\
Expertness & -0.09 & $-0.13^{*}$ & $-0.14^{*}$ \\
Attractiveness & $-0.12^{*}$ & -0.08 & $-0.13^{*}$ \\
Trustworthiness & $-0.12^{*}$ & $-0.14^{*}$ & $-0.16^{*}$ \\
Total & $-0.13^{*}$ & $-0.12^{*}$ & $-0.17^{*}$
\end{tabular}

*Indicates Significance at .05 level (Kendall’s Tau Correlation, 2-tailed)

Looking closer at the data in regards to the correlations between CERS and MCKAS scores across race, White youth at risk participants tended to indicate a very small negative relationship between counselor multicultural knowledge and overall competence with their levels of effectiveness. Further, ethnic minorities tended to indicate a very small negative relationship between counselor multicultural awareness with their levels of effectiveness. (see Table 12 and 13). 
Table 12

Correlations of CERS and MCKAS Scores for White Participants $(n=54)$

MCKAS

\begin{tabular}{llll}
\multicolumn{1}{c}{ CERS } & Knowledge & Awareness & Total \\
Expertness & $-0.14^{*}$ & -0.07 & $-0.18^{*}$ \\
Attractiveness & $-0.17^{*}$ & -0.08 & $-0.18^{*}$ \\
Trustworthiness & $-0.13^{*}$ & -0.09 & $-0.16^{*}$ \\
Total & $-0.19^{*}$ & -0.10 & $-0.22^{*}$
\end{tabular}

Table 13

Correlations of CERS and MCKAS Scores for Ethnic Minority Participants $\quad(\mathrm{n}=32)$ MCKAS

\begin{tabular}{llll}
\multicolumn{1}{c}{ CERS } & $\underline{\text { Knowledge }}$ & $\underline{\text { Awareness }}$ & $\underline{\text { Total }}$ \\
Expertness & -0.05 & $-0.20^{*}$ & -0.12 \\
Attractiveness & -0.05 & -0.07 & -0.07 \\
Trustworthiness & -0.11 & $-0.21^{*}$ & $-0.17^{*}$ \\
Total & -0.07 & $-0.15^{*}$ & -0.11
\end{tabular}

*Indicates Significance at .05 level (Kendall’s Tau Correlation, 2-tailed) 


\section{Research Questions}

The reader will recall that the research questions driving this study are as follows:

1. How does multicultural competence of adventure based counselors relate to ethnic minority and White youth at risk participant ratings of their counseling effectiveness?

2. Are the generated results of Research Question 1 due to multicultural counseling competence, or are they the result of general counseling competence?

An analysis of the data suggests that a very small negative relationship exists between multicultural competence of adventure based counselors and ratings of their counseling effectiveness by youth at risk participants. Although significant, counselor multicultural competence only explained $2.8 \%$ of the effectiveness rating variance. These negative correlations between the CERS and MCKAS run counter to the original hypothesis that higher levels of counselor multicultural competence should result in higher levels of effectiveness with ethnic minority clients.

Further analysis was unable to secure evidence to determine if multicultural and general counseling competence were considered to be separate constructs based on multicultural variables. The only observed trend was a very small negative relationship between counselor levels of effectiveness based on their levels of multicultural competence for the White participants. Multicultural competence explained $4.8 \%$ of the variance for their levels of effectiveness with White participants, and was not a significant factor for ethnic minority perceptions of counselor effectiveness. 


\section{Discussion}

This section of Chapter 4 discusses the meaning of study's results and how they relate to relevant literature, identifies its limitations, gives recommendations for future research, and makes recommendations for the field of adventure-based programming. Counselor Effectiveness

Due to the restricted range and skewness of the dependent variable the assumptions for parametric analyses were not met for the Counselor Effectiveness Rating Scale. Consequently, the primary data analysis was restricted to nonparametric and descriptive analysis for the Counselor Effectiveness Rating Scale and the original hypotheses of the study. Some inferential statistics were used for exploratory analysis of the Multicultural Knowledge and Awareness Scale outcomes, but these results were interpreted with caution due to the increased likelihood of type I error.

\section{Counselor Effectiveness}

The most outstanding feature of the present study is the counselor effectiveness scores being extremely high. Scores in this range indicate that both the ethnic minority and White youth at risk were exceptionally satisfied with the performance of the counselors in regards to their expertness, attractiveness, and trustworthiness. A review of 14 studies exploring the relationship between multicultural variables and levels of counselor competence, found only one study that had higher levels of counselor effectiveness ratings (Thompson \& Littrell, 1998). A collective examination of the remaining studies found that the effectiveness ratings ranged from 3.0 to 6.2 with most of the scores falling between 5 and 6. This suggests that scores on the CERS for 
multicultural research tends to result with mean scores on the high side of the scale, but the scores still tend to be less than the mean of 6.4 found in the current study.

It is highly likely that confounding variables inflated the counselor effectiveness ratings to some degree. Based on discussions with the coordinator of the program used in the study and the investigator's four years of experience with the program, a number of variables may be responsible for the extremely high scores.

Probably the most prominent confounding variable was administering the CERS at the very end of the course. Newes (2001) suggests that measurements in adventure based programming may adversely be impacted by "pre-trip emotionality or post-trip euphoria” (p. 95). Typically at the end of the course, the group of participants are experiencing a "high" due to having successfully completed the course and the prospect of returning to their home within the next day or two (Davis-Berman \& Berman, 1994). They have shifted out of a demanding and relatively aversive environment to one of modern day comforts. For instance, the counselor generally provide an all you can eat dinner, movies, and graduation ceremony for the students during this stage of the course. There seems to be a high probability that the general satisfaction and contentment of the youths influenced the counselor effectiveness ratings in a positive direction. No literature could be found to help substantiate this hypothesis, but common sense and personal experience support it. To avoid this, it is recommended that CERS forms be administered multiple times through the 30 day course.

Similarly, group stage development may also have been responsible. Groups are likely to have a higher level of satisfaction with the group during and after it reaches the “performing” stage, which tends to be in the later stages of group development (Corey, 
2000; Gladding, 1999). In contrast, the group is likely to rate their satisfaction lower when in the earlier stages of adjustment and "storming,” which tends to be marked by conflict, dominance, and rebellion (Gladding, 1999; Yalom, 1975). This stage is often times marked by a general dissatisfaction with the group and its facilitators (Gladding, 1999; Yalom, 1975). These groups are likely to hit the storming stages in the middle third of the course and have likely moved in to cohesion and general satisfaction by the time the questionnaires were administered (Yalom, 1975). It is highly likely that the participants' ratings of the counselors' levels of effectiveness would have been considerably lower if it had been collected at earlier times during the courses.

Another consideration for the high effectiveness ratings is that the field typically attracts healthy young adults who tend to be relatively adventurous, altruistic, and socially adept (Bruyere, 2002). It is probable that these characteristics made the counselors naturally likeable. In addition to this, living with persons for 30 straight days under challenging conditions typically results in very close bonding between the counselors and participants (Davis-Berman \& Berman, 1994; Gass, 1993). It is a reasonable possibility that the participants would rate counselors as highly effective based on some of these characteristics than solely on their general and multicultural counseling skills.

Some participants may have also rated counselors higher than their actual perception because of the possibility that the counselors could potentially have access to the results. The logistician overseeing the administration of the instruments could have also impacted the participants' responses because the logistician is oftentimes considered by the participants to be in a similar position as the course counselors. Many times the 
recommendations given by the counselors after completion of the course had considerable positive or negative consequences for the youths. Some of the participants may have been concerned about being adversely affected if the counselor or logistician viewed lower ratings.

Even with the likelihood these confounding factors inflating the participants’ ratings of counselor effectiveness, it can be said with relative confidence that the participants were generally very satisfied with the counselors' level of attractiveness, expertness, and trustworthiness.

\section{Counselor Multicultural Competence}

The main factors with multicultural competence examined were in regards to the relationship between the counselors' multicultural competence and their demographics. Overall, few significant outcomes were identified, and the ones that were identified should be interpreted with caution considering that they have a high probability of type I error due to the number of factors that were analyzed.

Counselors appeared to show a pattern of scoring higher on levels of knowledge in comparison to their levels of awareness. This may suggest that the counselors in the study tended to have more comprehension of the worldview, values, and challenges of culturally dissimilar clients in comparison to their understanding one's core assumptions, principles, and prejudices, based on one's own cultural heritage as well as perceptions of other cultural systems. No literature that addresses this issue could be located.

A finding that appears to be counterintuitive is found with the relationship between counselor multicultural competence and degrees sought by the counselors. The persons who scored lowest, social science majors, were the ones who likely would have 
had the most exposure to multicultural concepts and theories. A possible explanation is that the counselors with social science backgrounds knew more accurately what they didn’t know and/or they responded in ways that more accurately assessed their actual levels of multicultural competence.

\section{Relation Between Counselor Effectiveness and Multicultural Competence}

Correlations between counselor levels of effectiveness and multicultural competence suggest a very small negative relationship. Counselors who achieved higher scores on the multicultural competence instrument tended to be scored lower by the youth at risk participants. Analysis of the relationship between counselor effectiveness and multicultural competence suggests trends contrary to relevant literature. The results indicate, that as indices of counselor multicultural competence increases, their levels of effectiveness with the youth at risk participants decreases. This runs contrary to body of literature on counselor effectiveness and multicultural competence. The body of literature in this area has consistently determined that the higher the level of counselor multicultural competence the higher the level their effectiveness should be (RamosSanchez, Atkinson, \& Fraga, 1999; Ruelas, Atkinson, \& Ramos-Sanchez, 1998).

Closer inspection of the data across ethnicity found that White youth at risk participants tended to have stronger negative correlation between Knowledge and Total CERS ratings, while ethnic minorities tended to have stronger negative correlations between Awareness and CERS ratings. This suggest that counselors who were likely to have more comprehension of the worldview, values, and challenges of culturally dissimilar clients where rated less effective by White youth at risk, while counselors who were more understanding to one’s core assumptions, principles, and prejudices based on 
one's own cultural heritage as well as perceptions of other cultural systems were determined to be less effective by the ethnic minority youth at risk.

The number of findings that are contrary to related literature and common sense suggest that factors may have contributed to the negative relationships found between education, age, and ethnicity. Pope-Davis, Liu, Toporek, and Brittan-Powell (2001) suggest that consumers may actually interpret counselor multicultural competence differently from those in the profession. Some studies have shown that clients prefer certain counselor characteristics independent of their level of expertise and multicultural competence (Atkinson, Kim, \& Caldwell, 1998; Atkinson \& Wampold, 1982). For example, extraneous factors may be due to older more educated counselors not being able to relate to the youth at risk participants in a manner that was deemed more effective by the youths. It is quite possible that these factors overshadowed many of the subtle skills related to effective multicultural counseling (Coleman, 1998).

\section{Limitations of the Study}

The first area of limitation is related to concerns with measurement and instrumentation. The main limitation that the study encountered was due to the CERS range of scores being very constricted to the positive side of the scale. Due to the CERS being severely limited to its highest segment of scores, an inferential statistical analysis could not be conducted. It is likely that the CERS is either not sensitive enough to determine differences between counselors for this population or it was heavily influenced by extraneous factors noted earlier. A review of studies, using the CERS, tends to indicate that the CERS has a limited range of responses and that the respondents rate counselors on the high side of the scale (Thompson \& Littrell, 1998). 
The study may have also been adversely impacted by the MCKAS’s high face validity being susceptible to a social desirability affect. Constantine and Ladany (2000) suggest that self-report measures of multicultural competence have questionable validity due to the influence of social desirability. Further, some of the questions on the MCKAS that inquire about exposure to various multicultural theories, research, and training would likely be very low for this group of counselors since they collectively had little training in fields that address multicultural interventions. This implies that some counselors being honest about their levels of exposure would score on the low end of the scale, while counselors influenced by social desirability would rate themselves as high, even if they had very limited exposure.

Problems with sampling also limited the study. Although the current study accessed counselor and participants from a number of 30-day courses, the samples were drawn from only one adventure based program. As a result, the external validity and generalizability of the findings are considerably limited. Also, it is quite likely that the 11 youths who were removed from the courses would have had an impact on the results. It is probable that these participants would have rated the counselors at a lower level than the included sample because participant are typically removed due to behavioral and interpersonal problems. The study also did not have enough ethnic minorities counselors to accurately compare them to the White counselors on various characteristics. This prohibited the study from being able to investigate a significant relationship between counselor ethnicity and their multicultural competence.

The study also contains some research and methodological limitations. Some of the methodological issues are the current study's design does not include manipulation of 
independent variables, randomization, or control groups. Much of this was due to limitations inherent in using a field study with a quasi-experimental design. As a result, no causal relationships can be inferred.

Further, if the study had followed through with the original analysis with a MANOVA, it is likely that it would have violated the assumption of independence of individuals and groups. After spending 30 straight days with one another, it is extremely likely that the participants and counselors directly influenced how others responded on the measures. In addition, the variation among counselors would likely have been contaminated by one another because the clients’ response would have been pooled across counselors.

\section{Recommendations for Future Research}

The results, methodological issues, and interpretive considerations of the present investigation suggest the following recommendations for future research. Future research will benefit from using more appropriate instruments and data collecting procedures, utilizing qualitative analysis, using an instrument that directly assesses both consumer perceptions of counselor multicultural and general counseling competence, and investigating the impact that multicultural training has on levels of competence and effectiveness.

Due to the problems encountered in the present study, alternative instruments and forms of data gathering are recommended for future research. It is suggested that the typical surveys used for assessing counselor effectiveness not be used due to the CERS's high concurrent validity with other counselor effectiveness surveys (Pope-Davis \& Dings, 1994). Collecting the data through detailed interviews and other qualitative 
methods may help to avoid the restricted range of scores found in the current investigation (Pope-Davis, Liu, Toporek, \& Brittan-Powell, 2001).

Qualitative research also may be more successful at determining the effect that counselors' multicultural competence has on the youth at risks' experiences and yield more important descriptive data of effective multicultural interventions. In depth interviews with the participants and/or essays on their experiences with the individual counselors may illuminate more of the salient, subtle, and untapped features of their perceptions. Consensual Qualitative Research is suggested by Fuertes (2001) as a particularly productive way of attaining qualitative data by interviewing the client and counselor during or after treatment is completed. Detailed information acquired through this process can elicit rich information used to deepen our understanding of how multicultural issues play out in treatment and help to guide research, practice, and training.

Future researchers should consider administering a questionnaire that more specifically address the youths’ perceived level of multicultural competence of the counselors. Fuertes (2001) suggests that some of the current multicultural competence instruments may be modified and used in order to include the "client's voice." For instance, Fuertes and Brobst (2003) altered the Cross-Cultural Counseling InventoryRevised in a study to directly assess clients’ perceptions of their counselors’ multicultural competence. Fuertes goes on to suggests that it may be particularly beneficial to go beyond examining traditional counseling skills (e.g. expertness, attractiveness, trustworthiness) by investigating the variance in process and outcome variables 
specifically related to counselor multicultural competence (i.e. cultural understanding, racial mistrust, respect of diversity, value barriers).

It is also recommended that data be collected at various points of the course to avoid the possible confounding affect of group stage influence and program completion (Newes, 2001; Yalom, 1975). To get a more comprehensive sampling, the investigator recommends that data collection take place at least halfway through and at the end of the program.

As with other research investigating the relationship between ethnicity and counseling, future investigation may benefit from taking into consideration issues beyond merely race, including cultural makeup (i.e. socio-economic history, environmental characteristics, and socio-political influences), racial identity development, and cultural mistrust of both the youth and counselors. This could help better explain the complex aggregate of factors that impact multicultural issues and the process of counseling.

Consistent with the current study, researchers recommend using field studies and quasi-experimental designs in order to access real-life situations and practical information Fuertes, 2001; Pope-Davis, Liu, Toporek, \& Brittan-Powell, 2001). However, it is likely that many of the studies short-comings are directly related to doing a field study. It is recommended that future research control as many potential extraneous variables as possible and manipulate only key variables without interfering with the natural elements fundamental to the benefits of a field study.

The adventure based field also could benefit from literature determining effective forms of multicultural training. Pope-Davis and Dings (1995) addressed the need to examine and evaluate how multicultural training should be conducted and to evaluate the 
effectiveness of its content and format. An experiment could be devised that would evaluate various forms of multicultural training with the qualities of a field study and quasi-experimental design. This study could compare groups of counselors exposed to various multicultural training conditions with one another as well as with a control group of counselors who have not been exposed to training.

\section{Recommendations for the Field of Adventure Based Programming}

Due to the lack of consistent or statistically reliable findings of the study, the recommendations for the field of adventure based programming are largely based on literature examining the main factors of the study.

According to Warren (2002), there has been growing awareness of the need for multiculturally competent adventure based professionals, but little research or training material is available to meet this need. Research needs to further investigate the level of multicultural competence and its application by adventure based professionals as well as the impact that it is having on its ethnically diverse clientele. The paucity of research in this area implies that very little teaching and awareness of these issues exists in the training and evaluation of professionals.

Multicultural instruction and exposure should be included in all programs that serve youths of color. In her critique on traditional forms of outdoor leadership trainings, Warren (2002) noted that university programs, texts, field schools, and staff trainings for the adventure based treatment field were noticeably lacking in issues of diversity and marginalized groups. Considering the high number of ethnic minorities referred to adventure based programs, it would be beneficial to require programs serving ethnic minorities to have a multicultural element in their training. Multicultural training 
programs result in increased positive rating of counselor effectiveness by ethnic minority clients, greater expertise, trustworthiness, attractiveness, empathy, and unconditional positive regard (Wade \& Bernstein, 1991).

According to Constantine (2001) in nearly all studies investigating the relationship between levels of multicultural training and competence, it has been found that the training is significantly correlated with higher levels of competence in working with culturally diverse clients. Studies have indicated that multicultural instruction has been associated with increases in interracial comfort and White racial consciousness (Parker, Moore, \& Neimeyer, 1998), conceptualization and treatment efficacy of multicultural mental health issues (Constantine \& Ladany, 2000), and self-and other perceived multicultural competence (Holcomb-McCoy \& Myers, 1999).

Wright (1997) devised a multicultural education program that utilizes shared adventure as a means for increasing multicultural competence with outdoor professionals in training. His model consists of a diverse group of individuals participating in a shared/structured task that incorporates adventure and challenge. The activity is created so that group cooperation and support is essential for its successful completion. The group follows the activity by debriefing what took place and by using the experience as a vehicle for dialogue. The shared experiences and cooperation, essential to these activities, creates the opportunity for diverse individuals to connect on a deeper level than that typically found with traditional multicultural training methods. The resulting dialogue about cooperation and success as well as conflict and controversial issues that arise from the activities can create an opportunity for intra and interpersonal discovery. This corresponds with Meyer’s (1994) argument that multicultural training should go 
beyond “shallow expressions of tolerance and diversity” (p.11) and Welp’s (1997) belief that the only way for transformative learning to take place is by building friendships and alliances over differences.

In addition to training, the field needs to increase access and exposure to various cultural systems in order to more adequately meet the need of ethnic minorities. The imbalance of ethnic minority participants to adventure based counselors found in this study is likely a common element in the field of adventure based and other social services (Bernal \& Castro, 1994; Bruyere, 2003; Warren, 2002). One way to help balance this disproportion, and more effectively meet the needs of the ethnic minority clients in environmentally related fields, is by recruiting and hiring persons of color (Ashley, 1990; Benepe, 1992; Matthews, 1994). Increasing the number of ethnically diverse practitioners, researchers, and administrators in the field of adventure based programming may improve the level of care given to the ethnic minority youth by broadening the multicultural sensitivity and understanding of the adventure based programs, policies, and White counselors (Warren, 2002). Recruiting ethnic minority counselors and administrators and hiring more persons of color may be beneficial due to ethnic minority preference for and higher level of satisfaction with ethnically similar counselors (Warren, 1999).

Diversity would also be beneficial to the predominantly White staff. Research suggests that persons who have been exposed to groups and cultural environments different than their own become more multiculturally competent (Diaz-Lazar, 2001). Interacting with various cultural values, norms, and perspectives are important factors in broadening one’s knowledge and awareness of persons different from one’s self. 
According to Allport (1954), for successful understanding of persons from different cultures, it is necessary to go beyond didactic training by the individuals becoming personally involved. The extended contact, shared experience, and personal encounters that naturally take place between employees of adventure based programs would likely ensure that the conditions of the contact hypothesis successfully would be met for the ethnic minority and White employees (Wright, 1997). By increasing the diversity of staff and administrations through hiring practices, the field also would create opportunities for the inclusion of persons of diversity in the decision and policy making process. This is consistent with Warren's (1998) suggestion that programs and facilitators need to assess their policies and interventions from a multicultural framework and to adjust accordingly. She stresses that more important than techniques, facilitators need training in the "social and cultural backgrounds of their participants and the way their locations in privilege or marginality affect who they teach and facilitate” (p. 23).

One problem to consider is the paucity of persons of color participating in wilderness and adventure based activities in the United States (Warren, 1999). James and Cavy (1992) suggest working to increase interest, exposure, and opportunities of environmental activities for persons of color as a means of increasing the potential pool of diverse individuals who could be recruited into the field. This could be accomplished by adventure based associations and programs taking active steps to connect with groups of ethnic minorities and provide opportunities for them to participate in outdoor activities. Roberts (1996) suggests increased exposure by adventure based professionals going to ethnically diverse community settings to promote adventure based activities through mentoring and internship opportunities. Another hurdle to overcome is related to 
the economic imbalance for ethnic minorities and their ability to access adventure based activities and programs. Funding in this area could help reduce the "elitist nature” of the adventure based field by providing opportunities to ethnic minorities through grants, programs, and scholarships to assist them in gaining the experience and qualifications necessary to work in the field (Benepe, 1992).

Finally, it is also recommended that associations and accrediting agencies, such as the Association of Experiential Education, set a minimum standard for multicultural training and ethnic minority representation. It is hoped that making these changes would help the field more closely meet the ethical standards for working with multicultural clients as defined by the TAPG.

In conclusion, the field of adventure based programs need to make considerable changes in its training, hiring, research, and certifications in order to more effectively meet the needs of its ethnically diverse clientele. By doing this, counselors in the field, as well as the programs, will be more effective at helping the ethnic minority youth at risk return to the culturally complex worlds they come from as more productive members of their society. 


\title{
CHAPTER FIVE
}

\author{
Summary
}

\section{Youth at Risk}

Our society has significant investment in the health and well being of today's adolescents, but the rates of at risk behaviors for America's youth continue to increase at an alarming rate (Fields \& McNamara, 2003). Compared to White youth, ethnic minority youth participate at an even higher prevalence of activities and situations that are dangerous to their health and safety as well as that of others (Franke, 2000; Harvey \& Coleman, 1997; Stevens \& Griffin, 2001).

According to Franke (2000), an overwhelming number of today’s ethnic minority youth grow up in particularly impoverished and dangerous conditions that promote highrisk behaviors. Ethnic minorities youths are at an exceptionally high risk due to the exploitation, discrimination, and subordination they experience in addition to the pressures of adolescence (Howard-Hamilton \& Behar-Horenstein, 1995). These increased pressures adversely impact their sense of self, others, and the world (Davis, 1999) and contribute to their higher prevalence of antisocial and criminal behavior (Gullotta, 2000). Many of the "negative” behaviors youth at risk display are actually attempts at coping with their daily struggles inherent in their socio-cultural environment (McWhirter, McWhirter, McWhirter, \& McWhirter, 1993). Eventually, these survival skills, negative adaptive strategies, and search for meaning can manifest into destructive behavioral patterns (Capuzzi \& Gross, 1996). Tidwell and Garrett (1994) note that these behaviors and attitudes develop into a cumulative, self-fulfilling cycle of deviance. 
Considering the breadth of this problem, there is a noticeable lack of solid empirical literature on treating youth at risk (Dalton, 1998). Even with these theoretical and empirical limitations, many forms of treatment have been instituted to manage the problems that youth face as well as the problems they cause in society. The finding that "traditional" forms of treatment, including incarceration and talk therapy, have demonstrated to not be as effective as originally thought (Bruyere, 2002), indicates that alternative interventions need to be investigated, developed, and employed (Bazemore \& Terry, 1997).

Research has found that rehabilitation (Boone, 1994), group counseling (Goodman \& Getzbel, 1996; Zink \& Littrell, 2000), and interventions based on action and healthy risk (Albanese, 1993) are particularly effective at reducing the level of risk with adolescents. Adventure based interventions are an alternative approach to serving youth at risk that typically employs these characteristics that has earned consistent support (Buyere, 2002; Davis-Berman \& Berman, 1994). Although youth at risk, served through adventure based programming, is ethnically diverse, literature has also neglected to investigate how it relates to ethnic minorities (Bruyere, 2002; Liu \& Clay, 2002).

\section{Adventure Based Treatment}

Adventure based counseling, a form of intervention that concentrates on experiential-based learning to facilitate behavior change in the wilderness (Gass, 1993), is one manner of intervention that has shown merit and substantial development in treating youth at risk (Bandoroff, 1992). Adventure based programming was developed primarily on the concept that behavior change and deeper learning happen more effectively through direct experience (Kraft \& Sakofs, 1985). Adventure based treatment 
programs typically draw from these concepts in several ways. They create experiences that challenges persons to think and behave in novel and healthier ways (Kraft and Sakofs, 1985), encourages problem solving and critical thinking (Herbert, 1996), increases awareness of one's constructive as well as maladaptive patterns through direct experience (Gass, 1993), presents strong reinforcement properties and accountability through immediate and natural consequences (Russell, 2001), and motivates through the use of fun (Herbert, 1996).

Although youth at risk are not the only population that participates in programs that use adventure as a means of treatment, they do comprise the largest portion of the populations served (Davis-Berman \& Berman, 1993). The main goals for most of these programs are to help at risk youth replace self-destructive behaviors with healthier ones (Gass, 1993). A significant body of research exists that indicates that this type of intervention is an effective form of treatment for many issues that youth at risk present (Bruyere, 2002). Among many other problems that youth at risk face, studies have illustrated that adventure based programming has been effective with at risk youth in treating substance abuse (Gillis \& Simpson, 1991; Skafos, 1992), reducing recidivism rates (Newes, 2001; Sakofs, 1992; Wichmann, 1991), enhancing interpersonal skills (Herbert, 1996), treating mental illnesses (Kelley \& Selby, 1997), improving self-esteem (Moote \& Wodarski, 1997), and improving locus of control (Han, 2000).

Adventure based programming offers unique conditions and situations that make it particularly effective with youth at risk. Many of the programs take the youths out of unhealthy environments and put them into a wilderness setting where their past interaction styles become ineffective (Gass, 1993). The wilderness provides many 
opportunities for natural consequences, both positive and negative. Frequently, behaviors have direct consequences and the youths are reinforced immediately for their good and poor decision making (Davis- Berman \& Berman, 1994; Gass, 1993). Fletcher and Hinkle (2002) claim that the conditions and adventure based interventions allow youths the opportunity to change dysfunctional patterns of behavior and to redefine themselves. Adventure based programs are often devised to present progressive stressors and challenges to the youths (Davis-Berman \& Berman, 1994). Presenting the challenges in a progressive manner sets the youths up for success and helps them build a sense of accomplishment, improve self-esteem, understand the benefits of teamwork, and provide an opportunity for growth (Bacon \& Kimball, 1989; Davis-Berman \& Berman, 1994, Gass \& Gillis, 1993). These challenges also encourage the participants to "push their comfort zone” (Fletcher \& Hinkle, 2002). Davis-Berman \& Berman (1999) contend that some of the most significant growth occurs when participants are on the edge or go slightly beyond their comfort zone. When youths successfully do this, they typically are proud of their accomplishments, their comfort zone expands, and they become richer and healthier individuals. These challenges also present the opportunity for conflict to arise. During times of stress, many of the youths' dysfunctional patterns emerge which allows the adventure based counselors and the youths’ peers to work directly with many of the issues that have been putting the youths at risk (Wichmann, 1991).

Literature on adventure based programming as a whole claims to have consistent success with few negative outcomes in treating youth at risk (Burton, 1981; Friese, Pittman, \& Hendee, 1995). However, these “successes” are based on very small to medium outcomes with considerable variability between effect sizes (Neill, 2003). 
Recently there has been increasing pressure within the field to produce higher quality research (Davis-Berman, Berman, \&Capone, 1994; Neill, 2003; Newes, 2001) and to explore multicultural aspects. Particular areas that have been neglected within the field are accessing the characteristics of the facilitators and the impact that it has on its ethnic minority clients (Bruyere, 2002; Warren, 2002; Weston, Tinsley, \& O’Dell, 1999).

\section{Multicultural Competence}

Since the 1970's, social sciences have become more aware of the need for effective multicultural counseling application and research (Atkinson, Morten, \& Sue, 1993). The main force behind more multicultural competence was from the realization that the field of psychology had inadequately attempted to address culturally complex issues with culture-blind theory based predominantly on European American contexts (Atkinson et al., 1993). Pederson (1991) contends that multiculturalism is an essential and distinct component of effective counseling. He believes that to be effectual, counselors must go beyond general counseling skills by valuing and recognizing the impact that cultural influences have on the client and counselor as well as in their relationship.

Even in light of the gains over the past 30 years, leaders in the field of counseling believe that the majority of White counselors are not competent at conducting adequate assessment, research, and interventions with ethnic minorities (Bernal \& Castor, 1994; Malgady, 1996). In an attempt to improve understanding of and service to persons of color, professionals attempted to define multicultural counseling competencies and standards. In an attempt to better define these competencies and standards, Sue, Arrendondo, and McDavis (1992) distilled these concepts into three basic constructs: 
awareness, knowledge, and skill. It is believed that if counselors have adequate understanding and can effectively employ all three of these constructs that they are considered to be multiculturally competent counselors (Holcomb-McCoy \& Meyers, 1999). These three competencies have also been broadly accepted in the field of counseling and used as guidelines for training, research, and practice (Sue, Carter, Casas, Foad, Ivery, Jensen, LaFromboise, Manese, Ponterotto, \& Vasquez-Nutall, 1998).

The inquiry into multicultural competence has generally found ethnic minorities to be superior to Whites in multicultural counseling. The only demographic variable identified as a primary predictor of the level of counselor multicultural competence is ethnicity (Holcomb-McCoy \& Myers, 1999; Manese, 2001; Pope-Davis, Reynolds, Dings, \& Nielson, 1995). In particular, the body of literature in this area generally indicates that that ethnic minorities are more competent than Whites at working with people of color (Ponterotto, Rieger, Barrett, \& Sparks, 1994; Sodowsky, Kuo-Jackson, Richardson, \& Corey, 1998), and that whites overestimate their actual multicultural counseling abilities (Granello \& Wheaton, 1998; Pope-Davis \& Ottavi, 1994; Sue \& Zane, 1987).

This suggests that various factors related to the ethnicity and multicultural competence of counselors directly impacts their effectiveness with ethnic minority clients. Most research in this area has typically investigated perceived effectiveness based on Strong's (1968) theory of counselor influence. Strong hypothesized that the outcome of therapy is directly related to the degree that clients view therapists as being an expert, attractive, and trustworthy. In general, the three main constructs of this theory have been empirically validated through dozens of studies (Gelso \& Fretz, 1992), and 
have been utilized as dependent variables in several investigations on counselor effectiveness ratings as perceived by their ethnic minority clients (Pope-Davis and Dings, 1995). Investigation in this area is particularly important because the mental health needs of ethnic minorities have not been adequately met in application nor in research (Okonji \& Osokie, 1996).

In order to increase utilization, decrease premature termination, and improve services it is important that ethnic minorities perceive the counseling setting to be safe and the counselor to be a credible source of help for their issues (Pedersen, 1988; PopeDavis \& Dings, 1991). In their study on the role of culture and cultural techniques in psychotherapy, Sue and Zane (1987) stress perceived counselor effectiveness and credibility as the most significant qualities in working with ethnically diverse individuals. Counselor Effectiveness

Studies examining counselor ethnic similarity effect on client satisfaction and outcome of treatment is “equivocal” at best (Atkinson \& Masushita, 1991). According to Coleman, Wampold, and Casali (1995) the "particularist approach,” clients preferring clients from their own ethnic group, has not consistently been supported. Although literature in this areas is mixed and needs further investigation, the current review tended to find that the more recent studies showed that ethnic minorities preferred ethnically similar counselors (Atkinson \& Lowe, 1995; Coleman, Wampold, \& Casali), ethnically similar counselors to be more effective (Atkinson, 1983; Atkinson \& Lowe, 1995; Okonji \& Osokie, 1996), and multiculturally competent counselors to be more effective with ethnic minority clients (Coleman, 1998; Gim, Atkinson, and Kim, 1991; Pomales, Claiborn, and LaFromboise, 1986). 
The current study was designed to investigate the impact that adventure based counselors' multicultural competencies have on their effectiveness ratings by participants, particularly ethnic minority participants. The need for well-designed empirical research on adventure based programs, the inconsistent literature with ethnic minorities and perceived counselor effectiveness, and the need to understand multicultural competence make this a useful study. The study examined the relationship between participant ethnicity and counselor multicultural competence, as measured by counselor effectiveness.

\section{Selection of Participants}

The study collected data from a program that facilitates 30-day adventure based courses for youth at risk. The youth at risk participants were accessed from an adventure based program who’s participants were referred by probation officers, caseworkers, and by private social service agencies. The participating counselors were accessed from the staff of the adventure based program. Data was collected from 12 courses over the duration of a year. The study included 86 youth at risk, with 32 of them being defined as ethnic minorities. The study included 35 counselors, with 3 of them being defined as ethnic minorities.

\section{Instruments}

The Counselor Effectiveness Rating Scale, which is divided into four subcategories, measures the dependent variable of the study: Expertness, Attractiveness, Trustworthiness, and Total Effectiveness (Atkinson \& Carskaddon, 1975). With permission from the author, Donald Atkinson, the CERS was modified to be more specific to the setting, the adventure based counselors, and the participants completing 
the questionnaire. According to the author, such changes did not affect the validity or reliability of the instrument (Atkinson, 2001).

The independent variables for the study are the demographic variables of the youth at risk and counselor participants as well as the counselors' self-reported multicultural competencies. Demographic variables include such factors as age, ethnicity, education, and work experience. Multicultural competencies are measured by the Multicultural Counseling Knowledge and Awareness Scale (MCKAS) (Ponterotto, Gretchen, Utsey, Rieger, \& Austin 2000). The factors consist of two sub-scales, multicultural Awareness and Knowledge/Skill, and a total score.

\section{Procedures}

Prior to the course, during one of the three days of course preparation, the Program Coordinator distributed the research instruments to the course counselors during an activity, called "staff talks." At that time the course counselors completed the research instruments, and enclosed them in a tamper-proof envelope, addressed to the investigator. Based on statistical concerns and practicality, the counselors would only complete the MCKAS once even though some of them would participate in more than one course included in the study.

Prior to the courses, the investigator prepared participant packets that include a Participant Research Questionnaire and 3 CERS instruments for every youth at risk participant to rate each of the counselors individually. Each mailing included 12 packets per course. The logistician for each course was given the participants' packets and distributed them during the day prior to each participant's graduation from the course. At the time of distribution he or she read the Wilderness Questionnaire Script and 
Administration Directions (Appendix C and D). Once participant instruments were completed, they were enclosed in a tamper-proof envelope and mailed to the investigator. Results

The result of the youth at risk participant ratings of the counselors suggests an extremely high level of perceived effectiveness. The median score for the counselors' level of effectiveness was 6.4 on a 7 -point rating scale. Only $2 \%$ of the scores fell below the “average” category of 4 on the Counselor Effectiveness Rating Scale. Due to the restricted range of scores, the assumptions of parametric statistics were not met. As a result, nonparametric statistical analyses and descriptive analyses were used in analyzing data related to counselor effectiveness.

An analysis of the data suggests that a very small negative relationship exists between multicultural competence of adventure based counselors and ethnic minority as well as white participant ratings of their counseling effectiveness. White youth at risk participants reported, that as counselor multicultural knowledge and total multicultural competence increased, their perceived effectiveness decreased. Ethnic minorities reported, that as awareness and total multicultural competence levels increased for adventure based counselors, their perceived level of effectiveness decreased. Although significant, counselor multicultural competence only explained $2.8 \%$ of the effectiveness rating variance. These negative correlations between the CERS and MCKAS run counter to the original hypothesis that higher levels of counselor multicultural competence should result in higher levels of effectiveness with ethnic minority clients.

Further analysis was unable to secure evidence to determine if multicultural and general counseling competence was considered to be separate constructs based on 
multicultural variables. The only observed trend was a stronger negative relationship between counselor levels of effectiveness based on their levels of multicultural competence for the White participants. Multicultural competence explained 4.8\% of the variance for their levels of effectiveness with White participants, and was not a significant factor for ethnic minority perceptions of counselor effectiveness.

\section{Discussion}

The current study has various limitations that include problems with measurements and instrumentation, sampling, and methodological weaknesses. The CERS problems appear to be due to the limited range of scores, the issues with the MCKAS are likely attributed to it being self-report and its high face validity, and research and methodological limitations. The narrow sampling of data from only one adventure based program resulted in the external validity and generalizability of the findings being considerably limited. Some of the methodological issues are the current study's design does not include manipulation of independent variables, randomization, or control groups. Much of this was due to limitations inherent in using a field study with a quasi-experimental design. As a result, no causal relationships could have been inferred. Despite these limitations the findings of the study due have implications for future research.

The results, methodological issues, and interpretive considerations of the present investigation suggest the following recommendations for future research. Future research will benefit from using more appropriate instruments and data collecting procedures, utilizing qualitative analysis, using an instrument that directly assesses both consumer perceptions of counselor multicultural and general counseling competence, and 
investigating the impact that multicultural training has on levels of competence and effectiveness. Research also needs to further investigate the level of multicultural competence and its application by adventure based professionals as well as the impact that it is having on its ethnically diverse clientele. The paucity of research in this area implies that very little teaching and awareness of these issues exists in the training and evaluation of professionals.

The adventure based treatment field needs to include multicultural instruction and exposure in all programs that serve youths of color. In her critique on traditional forms of outdoor leadership trainings, Warren (2002) noted that university programs, texts, field schools, and staff trainings for the adventure based treatment field were noticeably lacking in issues of diversity and marginalized groups. The field needs to improve multicultural training, incorporate multicultural theory system of beliefs and practice, increase ethnically diverse professionals through hiring and opportunities for exposure, and for associations and accrediting agencies to set a minimum standard for multicultural training and ethnic minority representation. 


\section{References}

Abramowitz, S. I., \& Murray, J. (1983). Race effects in psychotherapy. In J. Murray \& P. R. Abramson (Eds.). Bias in Psychotherapy (pp. 215-255). New York: Praeger Press.

Abreu, J. M., \& Gabarain, G. (2000). Social desirability and Mexican American counselor preferences: Statistical control for a potential confound. Journal of Counseling Psychology, 47, 165-176.

Abreu, J. M., Gim-Chung, R. H., \& Atkinson, D. R. (2000). Multicultural counseling training: Past, present, and future directions. The Counseling Psychologist, 28, 641656.

Albanese, J. (1993). Dealing with Delinquency. Chicago: Nelson-Hall.

Allport, G. W. (1954). The nature of prejudice. Cambridge, MA: Addison-Wesley. American Psychological Association. (1992). Ethical principles of psychologists and code of conduct. American Psychologist, 47, 1597-1611.

American Psychological Association. (1993). Guidelines for providers of psychological services to ethnic, linguistic, and culturally diverse populations. American Psychologist, 48, 45-48.

Arredondo, P., Toporek, R., Brown, S., Jones, J., Locke, D. C., Sanchez, J., \& Sandier, H. (1996). Operationalization of the multicultural counseling competencies. Journal of Multicultural Counseling and Development, 24, 42-78.

Ashley, F. B. (1990). Ethnic minorities involvement with outdoor education. In J. C. Miles \& S. Priest (Eds.). Adventure Education. (pp. 369-373). State College, PA: Venture. 
Atkinson, D. R. (1983). Ethnic similarity in counseling psychology: A review of research. The Counseling Psychologist, 11, 79-92.

Atkinson, D. R. (1985). A meta-review of research on cross cultural counseling and psychotherapy. Journal of Multicultural Counseling and Development, 13, 138-153.

Atkinson, D. R. (2001). Personal communication, April 15, 2001.

Atkinson, D. R., \& Carskaddon, C. (1975). A prestigious introduction, psychological jargon, and perceived counselor credibility. Journal of Counseling Psychology, 22, 180-186.

Atkinson, D. R., Casas, A., \& Abreu, J. M. (1992). Mexican-American acculturation, counselor ethnicity and cultural sensitivity, and perceived counselor competence. Journal of Counseling Psychology, 39, 515-520.

Atkinson, D. R., Kim, B. S. K., \& Caldwell, R. (1998). Ratings of helper roles by multicultural psychologists and Asian American students: Initial support for the three-dimensional model of multicultural counseling. Journal of Counseling Psychology, 45, 414-423.

Atkinson, D. R., \& Lowe, S. M. (1995). The role of ethnicity, cultural knowledge, and conventional techniques in counseling and psychotherapy. In J. G. Ponterotto, J. M. Casas, L. A. Suzuki, \& C. M. Alexander (Eds.), Handbook of multicultural counseling (pp. 387-414). Thousand Oaks, CA: Sage.

Atkinson, D. R., Morten, G., \& Sue, D. W. (Eds.). (1993). Counseling American minorities: A cross-cultural perspective (4th ed.). Madison, WI: Brown and Benchmark. 
Atkinson, D. R., Ponce, F. Q., \& Martinez, F. M. (1984). Effects of ethnic, sex, and attitude similarity on counselor credibility. Journal of Counseling Psychology, 31, 588-590.

Atkinson, D. R., Poston, W. C., Furlong, M. J. \& Mercado, P. (1989). Ethnic group preferences of counselor characteristics. Journal of Counseling Psychology, 36, 6872.

Atkinson, D. R., Wampold, B. E., Lowe, S. M., Matthews, L., \& Ahn, H. (1998). Asian American preferences for counselor characteristics: Application of the BradleyTerry-Luce model to paired comparison data. The Counseling Psychologist, 26, 101-123.

Atkinson, D. R., Winzelberg, A., \& Holland, A. (1985). Ethnicity, locus of control for gamily planning, and pregnancy counselor credibility. Journal of Counseling Psychology, 32, 417-421.

Atkinson, D. R., \& Wampold, B. E. (1982). A comparison of the Counselor Rating Form and Counselor Effectiveness Rating Scale. Counselor Education and Supervision, 22, 25-36.

Bacon, S. \& Kimball, R. (1989). The wilderness challenge model. In R. D. Lyman (Ed.), Residential and inpatient treatment of children and adolescents. New York: Plenum Press.

Bandoroff, S. (1992). Wilderness family therapy: An innovative treatment approach for problem youth. Unpublished doctoral dissertation, University of South Carolina. Baruth, L. G., \& Manning, M. L. (2000). A call for multicultural counseling and middle schools. Clearing House, 73, 243-247. 
Bazemore, G. T., \& Clinton, P. (1997). Developing delinquent youths: A re-integrative model for rehabilitation and a new role for the juvenile justice system. Child Welfare, 76, 665-717.

Benepe, S. (1992). Racial and ethnic diversity in wilderness use and environmental education. Lander, WY: National Outdoor Leadership School.

Bennett, C. (1995). Preparing teachers for cultural diversity and national standards of academic excellence. Journal of Teacher Education, 46, 259-266.

Bernal, M. E., \& Castro, F. (1994). Are clinical psychologists prepared for service and research with ethnic minorities? American Psychologist, 49, 797-805.

Block, S. (1994). Policing an increasingly diverse America. FBI Law Enforcement Bulletin, 6, 24-27.

Boone, H. N. (1994). An examination of recidivism and other outcome measures. American Probation and Parole Association Perspectives, 12-18.

Bruyere, B. L. (2002) Appropriate benefits for outdoor programs targeting juvenile male offenders. Journal of Experiential Education, 25, 207-213.

Burton, L. M. (1981). A critical analysis and review of the research on outward bound and related programs. Dissertation Abstracts International, 42, 1581B. (University Microfilms No. 8122147).

Capuzzi, D., \& Gross, D. (1996). Prevention: An overview. In: Capuzzi, D. \& Gross, D. (Eds.). Youth at risk: A prevention resource for counselors, teachers, and parents (2nd ed.). (pp. 21-33) Alexandria, VA: American Counseling Association.

Cason, D., \& Gillis, H.L. (1994). A meta-analysis of outdoor adventure programming with adolescents. The Journal of Experiential Education, 17, 40-47. 
Casas, J. M., \& Miguel, S. (1993). Beyond questions and discussions, there is a need for action: A response to Mio and Iambs. Counseling Psychologist, 21, 233-270.

Chambless, D. L., \& Hollon, S. D. (1998). Defining empirically supported therapies. Journal of Consulting and Clinical Psychology, 66, 7-18.

Coleman, H. L. K. (1998). General and multicultural counseling competency: Apples and oranges. Journal of Multicultural Counseling and Development. 26, 147-156.

Coleman, H. L. K., Wampold, B. E., \& Casali, S.B. (1995). Ethnic minorities’ ratings of ethnically similar and European American counselors: A meta-analysis. Journal of Counseling Psychology, 42, 55-64.

Constantine, M. G. (2001). Theoretical orientation, empathy, and multicultural counseling competence in school counselor trainees. Professional School Counseling, 4, 342-348.

Constantine, M. G. (2002). Predictors of satisfaction with counseling: Racial and ethnic minority clients' attitudes toward counseling and ratings of their counselors' general and multicultural counseling competence. Journal of Counseling Psychology, 49, 255-263.

Constantine, M. G., \& Ladany, N. (2000). Self-report multicultural counseling competence scales: Their relation to social desirability attitudes and multicultural case conceptualization ability. Journal of Counseling Psychology, 47, 155-164.

Corey, G. (2000). Theory and practice of group counseling (5th ed.). Pacific Grove, CA: Brooks/Cole.

Dalton, G. (1998). Poverty, single-parent households, and youth at-risk behavior: An empirical study. Journal of Economic Issues, 32, 1079-1105. 
Davis, N. J. (1999). Youth crisis: growing up in the high-risk society. Westport, CT: Praeger.

Davis-Berman, J., \& Berman, D. (1994). Wilderness Therapy. Dubuque, Iowa: Kendall Hunt.

Davis-Berman, J., Berman, D., \& Capone, J. (1994). Therapeutic wilderness programs: A national survey. Journal of Experiential Education, 17, 49-52.

Dryfoos, J. G. (1990). Adolescents at risk: Prevalence and prevention. New York: Oxford University Press.

Fields, S. A., \& McNamara, J. R. (2003). The prevention of child and adolescent violence. A review. Aggression \& Violent Behavior, 8, 61-91.

Fletcher, T., \& Hinkle, J. S. (2002) Adventure Based Counseling: An Innovation in Counseling Journal of Counseling \& Development, 80, 277-286.

Franke, T. (2000). Adolescent violent behavior: an analysis across and within racial/ethnic groups. Journal of Multicultural Social Work, 8, 47-70.

Friese, G., Pittman, J., \& Hendee, J. (1995). Studies of the use of wilderness for personal growth, therapy, education, and leadership development: An annotation and evaluation. Moscow, ID: University of Idaho Wilderness Research Center.

Fuertes, J. N. (2001). Future research directions in the study of counselor multicultural competency. Journal of Multicultural Counseling and Development, 29, 3-13.

Fuertes, J., \& Brobst, K. (2002) Clients' Ratings of Counselor Multicultural Competency. Cultural Diversity and Ethnic Minority Psychology, 8, 214-233.

Gass, M.A. (Ed.). (1993). Adventure therapy: Therapeutic applications of adventure programming. Dubuque; Iowa: Kendall Hunt. 
Gelso, C., \& Fretz B. (1992). Counseling Psychology. Orlando, FL: Harcourt Brace.

Gillis, H. L. (1992). Therapeutic uses of adventure-challenge-outdoor-wilderness: Theory and research, 35-47. Paper presented at the meeting of the Association for Experiential Education.

Gillis, H. (1995). If I conduct outdoor pursuits with clinical populations, am I an adventure therapist? Leisurability, 22, 5-15.

Gillis, H. L., \& Simpson, C. (1991). Project choices: Adventure-based residential drug treatment for court-referred youth. Journal of Addictions and Offender Counseling, $12,12-27$.

Gim, R. H., Atkinson, D. R., \& Kim, S. J. (1991). Asian-American acculturation, counselor ethnicity and cultural sensitivity, and ratings of counselors. Journal of Counseling Psychology, 38, 57-62.

Gladding, S. (1999) Group work: A counseling specialty (3rd ed.). New York: Merrill.

Glasser, (1993). Reality therapy, a new approach to psychology. In Gass, M.A. (Ed.). Adventure therapy: Therapeutic applications of adventure programming. Dubuque; Iowa: Kendall Hunt.

Gomez, R. A. (1991). Teaching with a Multicultural Perspective. ERIC Digest. (ED339548).

Goodman, H. \& Getzgel, G. (1996). Group work with high-risk urban youth. Social Work, 41, 375-382.

Granello, D. H., \& Wheaton, J. E. (1998). Self-perceived multicultural competencies of African American and European American vocational rehabilitation counselors. Rehabilitation Counseling Bulletin, 42, 2-16. 
Grieger, I., \& Ponterotto, J. G. (1995). A framework for assessment in multicultural counseling. In Ponterotto, J. G., Casas, J. M., Suzuki, L. A., \& Alexander C. M. Handbook of multicultural counseling. Thousand Oaks, CA: Sage.

Greene, B. A. (1986). When the therapist is White and the patient is Black: Considerations for psychotherapy from feminist heterosexual and lesbian communities. Women and Therapy, 5, 41-65.

Gullotta, T. (2000). The adolescent experience. San Diego, CA: Academic Press

Han, T. A. (2000). A meta-analysis of the effects of adventure programming on locus of control. Journal of Contemporary Psychology, 30, 33-60.

Harvey, A. R., \& Coleman, A. (1997). An Afrocentric program for African American males in the juvenile justice system. Child Welfare, 76, 197-204.

Hattie, J., Marsh, H.W., Neill, J. T., \& Richards, G. E. (1997). Adventure education and Outward Bound: Out of class experiences that make a lasting difference. Review of Educational Research, 67, 48-87.

Helms, J. E. (1984). Toward a theoretical model of the effects of race on counseling: A Black and White model. The Counseling Psychologist, 12, 153-165.

Herbert, J. T. (1996). Use of adventure-based counseling programs for persons with disabilities. Journal of Rehabilitation, 62, 3-9.

Hess, R. S., \& Street, E. M. (1991). The effect of acculturation on the relationship of counselor ethnicity and client ratings. Journal of Counseling Psychology, 38, 71-75.

Holcomb-McCoy, C. C. (2001). Exploring the self-perceived multicultural counseling competence of elementary school counselors. Professional School Counseling, 4, 195-202. 
Holcomb-McCoy, C. \& Myers, J. E. (1999). Multicultural competence and counselor training: A national survey. Journal of Counseling and Development, 77, 294-302.

Hovland, C. I., Janis, I. L. \& Kelley, H. H. (1953). Communication and persuasion: Psychological studies of opinion change. New Haven, CT: Yale University Press.

Howard-Hamilton, M. F., \& Behar-Horensteir, L. S. (1995). Counseling the African American male adolescent. Elementary School Guidance and Counseling, 29, 198206.

Hunt, J.S. (1994). Ethical issues in experiential education. Dubuque, Iowa: Kendall Hunt Publishing.

James, T. (1980). Sketch of a moving spirit: Kurt Hahn. Journal of Experiential Education, 3, 17-22.

Kelley, M. R, Coursey, R. D., \& Selby, R M. (1997). Therapeutic adventures outdoors: A demonstration of benefits for people with mental illness. Psychiatric Rehabilitation Journal, 20, 61-73.

Kerr, A. W., Hall, H. K., \& Kozub (2002). Doing Statistics with SPSS. Thousand Oaks, CA: Sage Publications.

Kielsmeier, J. (1989). Growing with the Times: A challenge for experiential education. The Journal of Experiential Education, 12, 12-17.

Kraft, R., \& Sakofs, M. (1985). The theory of experiential education. Boulder, CO: Association for Experiential Education.

López, R. S., López, A. A., \& Fong, K. T. (1991). Mexican Americans' initial preferences for counselors: The role of ethnic factors. Journal of Counseling Psychology, 38, 487-496. 
Liu, W. M. \& Clay, D. L. (2002). Multicultural counseling competencies: Guidelines in working with children and adolescents. Journal of Mental Health Counseling, 24, 117-118.

Malgady, R. G. (1996). The question of cultural bias in assessment and diagnosis of ethnic minority clients: Let’s reject the null hypothesis. Professional Psychology: Research and Practice, 27, 73-77.

Manese, J. E. (2001). The effect of training on multicultural counseling competencies: An exploratory study. Journal of Multicultural Counseling and Development, 29, 3141.

Matthews, B. (1994). Does outdoor and environmental education have a role in multicultural education? Taproot, Newsletter of the Coalition for Education in the Outdoors, Winter, 8-13.

Matthews, M. (1991). Wilderness programs offer promising alternatives for some youth: More regulation likely. Youth Law News, 12, 12-15.

McWhirter. J., McWhirter. B., McWhirter A., \& McWhirter E. (1993). At-risk youth: A complete response. Pacific Grove, CA: Brooks and Cole.

Meyer, M. A. (1994). A cultural imperative for wilderness adventure programs: A native Hawaiian example. Journal of Experiential Education, 17, 11-15.

Miner, J. L., \& Boldt, J. (1981). Outward bound U.S.A. New York: William Morrow. Mio, J., \& Iwamasa, G. (1993). To do, or not to do: That is the question for white crosscultural counseling. Counseling Psychologist, 21, 197-213.

Mitten, D. (1994). Ethical considerations in adventure therapy: A feminist critique. Women and Therapy, 15, 55-84. 
Moote, G. T., \& Wodarski, J. S. (1997). The acquisition of life skills through adventurebased activities and programs: A review of the literature. Adolescence, 32, 143-167.

Myers, L. B. (2000). Meeting correctional offender needs: An ethical response to cultural differences. Prison Journal, 80, 184-210.

Newes, S. L. (2001) Future Directions in Adventure-Based Therapy Research: Methodological Considerations and Design Suggestions, Journal of Experiential Education, 24, 2 92-100.

Newes (2001). Predicting recidivism among juvenile offenders participating in an adventure-based therapy intervention. Unpublished dissertation, The Pennsylvania State University.

Noguera, P. (2003). City schools and the American dream: reclaiming the promise of public education. New York: Teachers College Press.

Okonji, J. M. A. \& Osokie, J. N. (1996). Preferred style and ethnicity of counselors by African American males. Journal of Black Psychology, 22, 329-340.

Parker, W. A., Moore, M. A., \& Neimeyer, G. J. (1998). Altering White racial identity and interracial comfort through multicultural training. Journal of Counseling and Development, 76, 302-310.

Pedersen, P. B. (1988). A handbook fore developing multicultural awareness. Alexandria, VA: American Association for Counseling and Development. Pedersen, P. B. (1991). Multiculturalism as a generic approach to counseling. Journal of Counseling and Development, 70, 6-12. 
Pomales, J., Claiborn, C. D., \& LaFromboise, T. D. (1986). Effects of Black students' racial identity on perceptions of White counselors varying in cultural sensitivity. Journal of Counseling Psychology, 33, 57-61.

Ponce, F. Q., \& Atkinson, D. R. (1989) Mexican-American acculturation, counselor ethnicity, counseling style, and perceived counselor credibility. Journal of Counseling Psychology, 36, 203-208.

Ponterotto, J. G., Casas, J. M., Suzuki, L. A., \& Alexander C. M. (Eds.). (1995). Handbook of Multicultural Counseling. Thousand Oaks, CA: Sage.

Ponterotto, J. G., \& Furlong, M. J. (1985). Evaluating counselor effectiveness: A critical review of rating scale instruments. Journal of Counseling Psychology, 32, 597-616.

Ponterotto, J. G., Gretchen, D., Utsey, S. O., Rieger, B. P. \& Austin, R. (2000). A construct validity study of the Multicultural Counseling Awareness Scale (MCAS). Unpublished manuscript.

Ponterotto, J. G., Rieger, B. P., Barrett, A., \& Sparks, R. (1994). Assessing multicultural counseling competence: A review of instrumentation. Journal of Counseling and Development, 72, 316-322.

Pope-Davis, D., \& Dings, J. G. (1995). In Ponterotto, J. G., Casas, J. M., Suzuki, L. A., \& Alexander C. M. Handbook of multicultural counseling. Thousand Oaks, CA: Sage. Pope-Davis, D. B., Liu, W. M., Toporek, R. L., \& Brittan-Powell, C. S. (2001). What's missing from cross-cultural competency research: Review, introspection, and recommendations. Cultural Diversity and Ethnic Minority Psychology, 7, 121-138. 
Pope-Davis, D. B,. \& Ottavi, T. M. (1994). The relationship between racism and racial identity among White Americans: A replication and extension. Journal of Counseling and Development, 72, 293-297.

Pope-Davis, D. B., Reynolds, A. L., Dings, J. G., \& Nielson, D. (1995). Examining multicultural counseling competencies of graduate students in psychology. Professional Psychology: Research and Practice, 26, 322-329.

Poussaint, A. F. (2000). Lay my burden down: unraveling suicide and the mental health crisis among African-Americans. Boston: Beacon Press

Ramos-Sanchez, L., Atkinson, D. R., \& Fraga, E. D. (1999). Mexican American’s bilingual ability, counselor, bilingualism cues, counselor ethnicity, and perceived counselor credibility. Journal of Counseling Psychology, 46, 125-131.

Ridley, C. R. (1989). Racism in counseling as an aversive behavioral process. In P. B. Pedersen, J. G. Draguns, W. J. Lonner, \& J. E. Trimble (Eds.), Counseling across cultures (pp. 55-77). Honolulu, HI: University of Hawaii Press.

Roberts, N. S. (1996). Women of color in experiential education: Crossing cultural boundaries In K. Warren (Ed.), Women's voices in experiential education. Dubuque, IA: Kendal/Hunt.

Roberts, N. S., \& Rodriguez, D. A. (1999). Multicultural Issues in Outdoor Education. ERIC Digest. (ED438151).

Rosol, M. (2000), Wilderness therapy for youth at risk. Parks \& Recreation, 35, 9, 32-50.

Ruelas, S. R., Atkinson, D. R., \& Ramos-Sanchez, L. (1998). Counselor helping model, participant ethnicity and acculturation level, and perceived counselor credibility. Journal of Counseling Psychology, 45, 98-103. 
Russell, K. C. (2001), What is Wilderness Therapy? Journal of Experiential Education, 24, 70-80.

Sakofs, M. (1992) Assessing the Impact of the Wilderness Alternative for Youth Program: An Outward Bound Program for Adjudicated Youth. Journal of Adventure Education and Outdoor Leadership, 9, 16-21.

Smith, M. L. \& Glass, G. V. (1977). Meta-analysis of psychotherapy outcome studies. American Psychologist, 32, 752-760.

Sodowsky, G. R. (1991). Effects of cultural consistent counseling tasks on American and international student observers' perception of counselor credibility: A preliminary investigation. Journal of Counseling and Development, 69, 253-256.

Sodowsky, G. R., Kuo-Jackson, P. Y., Richardson, M. F. \& Corey, A. T. (1998). Correlates of self-reported multicultural competencies: Counselor multicultural social desirability, race, social inadequacy, locus of control racial ideology, and multicultural training. Journal of Counseling Psychology, 45, 256-264.

Spectrum Wilderness Programs. (2004). Webpage. Retrieved February 5, 2004 from http://www.pso.siu.edu/tonec/?spectrum

Stevens, P. \& Griffin, J. (2001). Youth High-Risk Behaviors: Survey and Results. Journal of Addictions \& Offender Counseling, 22, 31-47.

Stich, T. F., \& Gaylor, M. S; (1983). Outward Bound: An innovative patient education program. (ERIC Document Reproduction Service, ED247047)

Strong, S. R. (1968). Counseling: An interpersonal influence process. Journal of Counseling Psychology, 15, 215-224. 
Stutzner-Gibson, D., Koren, P., \& DeChillo, N. (1995). The youth satisfaction questionnaire: What kids think about services. Families in Society, 8, 616-624.

Sue, D. W., Arredondo, P. \& McDavis, R. J. (1992). Multicultural counseling competencies and standards: A call to the profession. Journal of Counseling and Development, 70, 477-486.

Sue, D. W., Bernier, J., Durran, A., Feinberg, L., Pedersen, P., Smith, E. \& VasquezNuttal, E. (1982). Position paper: Cross cultural counseling competencies. The Counseling Psychologist, 10, 45-52

Sue. D. W., Carter, R. Casas. J. M. Fouad. N., Ivey, A. E. Jensen. M., LaFromboise, T., Manese, J. E., Ponterotto, J. G. \& Vasquez-Nutall, E. \{1998). Multicultural counseling competencies: Individual and organizational development. Thousand Oaks. CA: Sage.

Sue, S. \& Zane, N. (1987). The role of culture and cultural techniques in psychotherapy. American Psychologist, 42, 37-45.

Therapeutic Adventure Professional Group, Association of Experiential Education (2002). Webpage. Retrieved February 5, 2004, from http://www.geocities.com/aee_tapg/tapg.html

Thompson, R. \& Littrell, J. M. (1998) Brief counseling for students with learning disabilities.. Professional School Counseling, 2, 60-68.

Tidwell, R. \& Garrett, S. C. (1994). Youth at risk: In search of a definition. Journal of Counseling and Development, 72, 444-446.

Tyler, F. B., Brome, D. R. \& Williams, J. E. (1991). Ethnic validity, ecology, and psychotherapy: A psychosocial competence model. New York: Plenum 
U.S. Bureau of the Census. (1995). Statistical abstracts of the U.S. (115th ed.). Washington, DC: Author.

Utts, J. M. \& Heckard, R. F. (2004). Mind on Statistics. Belmont; CA: Brooks/Cole.

Vinson, T. S. \& Neimeyer, G. J. (2000). The relationship between racial identity and development and multicultural counseling competency. Journal of Multicultural Counseling and Development, 2, 177-193.

Wade, P. \& Bernstein, B. L. (1991). Culture sensitivity training and counselor's race: Effects on Black female clients' perceptions and attrition. Journal of Counseling Psychology, 38, 9-15.

Warren, K. (1999). Unpacking the knapsack of outdoor experiential education: Race, gender, and class sensitive outdoor leadership. Unpublished doctoral dissertation, Graduate School of The Union Institute, Cincinnati, Ohio.

Warren, K. (2002). Preparing the Next Generation: Social Justice in Outdoor Leadership Education and Training. Journal of Experiential Education, 25(1), 231-239.

Watkins, C. \& Terrell, F. (1998). Mistrust level and its effects on counseling expectations in Black client-White counselor relationships: An analogue study. Journal of Counseling Psychology, 35, 194-197.

Weston, R, Tinsley, H.A., O'Dell, I, (1999) Wilderness Adventure Therapy For At-risk Youth, Parks \& Recreation, 34, 30-40.

Watkins, C. E., Terrell, F., Miller, F. S., \& Terrell, S. L. (1989). Cultural mistrust and its effects on exepectational variables in black client-white counselor relationships. Journal of Counseling Psychology. 36, 447-450. 
Welp, M. G. (1997). Pathways to diversity for white males: A study of white males' learning experiences of the path toward advocating for inclusion and equity. Dissertation Abstracts International, 58-10A, 3813.

Wichmann, T. (1991). Of Wilderness and circles: Evaluating a therapeutic model for wilderness programs. Journal of Experiential Education, 14, 43-48.

Wright, A. (1997). Multicultural education through shared adventure. Journal of Outdoor Education, 27, 4-15.

Yalom, I.: The Theory and Practice of Group Psychotherapy. New York: Basic Books, 1975.

Youngs, A. \& Novas, A. (1995). Accelerated learning: A new approach to cross-cultural training. FBI Law Enforcement Bulletin, 64, 14-17.

Zhang, N; Dixon, D. N. (2001) Multiculturally Responsive Counseling: Effects on Asian Students' Ratings of Counselors. Journal of Multicultural Counseling \& Development, 29, 253-263.

Zinck, K. \& Littrell, J. M. (2000). Action research shows group counseling effective with at-risk adolescent girls. Professional School Counseling, 4, 50-60. 


\section{Appendices}

\section{Appendix A}

Description of the Thirty Day Wilderness Therapy Program. The 30-day course is divided into five stages: immersion, expedition, solo, final expedition, and transition. The immersion phase, the first 10 to 12 days of the course, consists primarily of acclimating the youth to a wilderness environment, teaching them needed skills, and building group cohesion. The immersion phase takes place on or near the program's property. Expedition, the second major phase, occurs during the 15 to 17 days that follow immersion. During the expedition phase, the group travels long distances in vans to remote wilderness areas. The vast majority of the time is occupied with hiking, canoeing, and general camping activities. Towards the end of the expedition, the participants will spend two to three days by themselves on a solo experience. During the final expedition, the group of participants function autonomously under less control and supervision by the counselors. The final stage, transition, occurs during the final two or three days of the course. The goals of the transition stage are to re-acclimate the participants to society and to provide activities and discussion to help the participants retain the gains they have made.

On the first day of the course, program staff pick up the participants from their hometowns and drive them to a wilderness setting at or near Touch of Nature (TON) property. After being dropped off, the staff go through each participant's belongings with them to determine what they need to take for the immersion phase. The participants are debriefed about what is going to be happening in the near future and then go to sleep in a tent or under a tarp for shelter. All wilderness equipment will be provided by the 
program, including a large backpack that the participants can carry their clothes, food, belongings, and sleeping bag.

On the second day, the counselors spend time instructing the participants on the wilderness and camping skills they will be using for the next 29 days. Each participant is given a responsibility/chore (e.g. navigator, cook, leader of the day, etc.) that are assigned to them every day on a rotating basis. As with most days of the course, the counselors and participants will break camp in the morning and then hike or canoe a distance until they reach a destination to camp for the night. Normally, the counselors facilitate a psycho-educational or therapy group for about an hour every evening. The topics of group typically are suited to the phase of the course, the participants' needs, or the events that happened during the day. The counselors act as facilitators and all members are encouraged to actively participate. The groups often are dynamic and run with a loose structure.

The bulk of three days during immersion (stage 1) is devoted to group and individual challenge activities. These activities usually consist of rock climbing, participating in team's course activities, and partaking in activities on a high-ropes course. The activities are designed to challenge the youths and get them to work as a cohesive and supportive unit. All activities are based on the challenge by choice concept, in which participants will not be coerced to participate, nor will the choice of nonparticipation have a negative impact on their evaluation by the counselors. The majority of the other days during immersion are spent acclimating the participants to a wilderness environment, teaching them the skills needed for wilderness travel, and working though personal and interpersonal problems. The main forum for working through these 
problems is through the circles and the therapy groups held in the evening. It is essential that the individuals in the group learn basic camping and wilderness skills, and display some sense of stability and cohesiveness for them to progress to the expedition phase.

Between the immersion and expedition phases, the staff and participants clean their gear, pack food, organize equipment, and make preparations for the expedition. A mid-course evaluation is completed during this time period that is discussed with the participant and sent to the participant's referring agent. The participants are allowed to take showers and exchange some of their clothing.

The first part of the expedition stage (stage 2) involves spending between 10 and 24 hours in a 15-passenger van. Very few stops are made and the participants are always within view of the staff to keep negative behaviors to a minimum. Once the group arrives at their wilderness destination, they unload the vehicle and prepare for a trek. The treks usually consist of paddling canoes around 100 miles and portaging between lakes or hiking around 60 miles. The terrain and mode of travel varies with the time of year. The summers typically consists of canoeing in Canada, spring and fall consist of hiking and canoeing in Arkansas, and the winters consist of hiking in Texas.

The solo (stage 3) lasts between two and three days and takes place around the $22^{\text {nd }}$ day of the course. The solo consists of the participant remaining by him or herself in a set area devoid of human contact. The staff spend adequate time preparing the participants for this experience and give them numerous challenges and activities as well as a journal to complete. They are given a very small but adequate amount of food, water, shelter, and an emergency whistle. The staff check on each participant two to 
three times per day to give medication, make sure they are safe, and meet any necessary need he or she has. The solo is designed to encourage introspection and self-reflection.

During the final two days of expedition, some of the higher functioning groups are allowed to operate more autonomously on a final expedition (stage 4). Although under staff supervision, the group is able to function as if they were completing the trek and all daily activities independent of the counselors. This portion of the course typically only happens in a third of the courses.

During the transition stage (stage 5), the staff attempt to prepare the participants to productively return to their home environment and re-acclimate to society. A significant amount of time is spent debriefing and processing the experience as well as discussing what it will be like to return to their homes, schools, and other environments from which they came. The staff take time to evaluate each member individually and discuss his or her progress over the course. The counselors also conduct a group that encourages the members to give feedback to one another as well as the counselors. A large focus of transition is helping the participants retain the gains they have made and to make healthy plans for the future. The participants also clean and check in their gear, clean and repair equipment, and take a showers.

On the $29^{\text {th }}$ day of the course, a graduation ceremony takes place for the participants who successfully complete the course. Typically one in 10 participants do not successfully complete the course. The graduation typically consists of a dinner at an all-you-can-eat buffet. After the dinner, diplomas and awards are given out to the participants. The $30^{\text {th }}$, and final, day consists of driving the participants to their homes throughout the state of Illinois. 
After the course, the counselors write evaluations of the participants and make recommendations to the referring agents. About a week after the course, the staff drive around the state to conduct follow up meetings with participants, their families, and referring agents. It is common for these evaluations and follow up meetings to have significant positive or negative consequences for the participants.

The staff of each course was comprised of three counselors and one logistician. The counselors' duties included: running psycho-educational and therapeutic groups, organizing the course structure, leading all the phases of the course, providing first aid, conducting wilderness activities, teaching wilderness and camping skills to the participants, providing transportation, writing evaluations, conducting follow-up meetings with the participants, their families, and referring agents, and many other general duties.

The primary duty of the logistician was to support for the counselors and participants. This support typically included: re-supplying food and water, transportation, 24 hour emergency support, preparing wilderness equipment, and acting as a liaison between the counselors and coordinators. The logisticians typically had limited contact with the participants.

The three counselors lived with the same participants 24 hours a day for the full 30 days. The vast majority of this time was spent in a wilderness area, and was little interaction with the public and society. The counselors' duties were demanding due to meeting a large portion of the participants’ needs for over 700 straight hours. 


\section{Appendix B}

Theoretical Underpinnings of the Wilderness Therapy Program. According to

Wichmann (1991), the following is utilized in SW, “wilderness environment; primary peer group; stress and perceived risk; problem solving; a humanistic style of instruction; challenge, mastery, and reflection; and some form of reality therapy” (p.45). Wichmann proceeds to state that much of the SW's theoretical base for therapeutic intervention rests with Glasser’s (1993) reality therapy. Staff trainings typically incorporate a workshop on using the concepts of reality therapy with youth at risk in a wilderness context. These workshops are comprised of didactic instruction followed by experiential exercises where the staff are challenged to use interventions based on reality therapy constructs.

Wichmann (1991) believed that with SW programming a large enough peer group needs to be created to generate complex interpersonal issues. The role of the counselors is to promote an atmosphere of safety and challenge so that the participants can grow in a positive direction. It is also common for the counselors and participants to build strong bonds due to the amount of time they spend together and because of the many shared intense experiences. The wilderness environment often acts as a powerful agent of reinforcement. In the wilderness, natural consequences are common; however, Wichmann suggested the use of "circles" in the absence of natural consequences. Both counselors and participants have the option of "calling a Circle" to deal with problems that arise. The counselors and participants must stand in a circle until the interpersonal problem is solved. If the group successfully resolves the interpersonal problem, then interpersonal bonds are strengthened and stress is reduced; however, if the issue is not resolved then interpersonal stress and discomfort will result. According to Wichmann, 
"The ultimate goal is to teach students to run a Circle and solve interpersonal problems with little or no input by the instructors” (p.46). Wichmann outlined his model and gave a nice example of how a Circle might be run in his article. 


\section{Appendix C}

\section{Wilderness Participant Research Questionnaire}

The following questionnaire will be used for research purposes only. It is voluntary and will in no way affect your performance ratings. No one other than the researcher and his supervisor will view your completed questionnaire. Neither the staff members nor your referring agent will have access to how you have answered the questionnaire.

The first two pages request information about you. The following three pages are questionnaires that ask questions about the leaders of your course. Please look at the top of these pages to identify the counselor you are responding to for that particular questionnaire. You should complete on page per counselor.

After you have completed the questionnaires, please put them in the addressed tamperproof envelope by the administrator. Once all of the participants have completed the questionnaires and they have been enclosed in the envelope, the envelope will be sealed. The envelope will be put in the mail as soon as is reasonably possible and will only be opened by the principal investigator.

Please answer the questions to the best of your ability. If you have any questions or concerns, please share them with the person administering the questionnaires. Thank you for your help.

What is your age?

What is your gender?

What race are you? (Please check one)

$\begin{array}{lr}\text { African American } & \text { Asian American } \\ \text { Hispanic/Latino/Latina American } & \text { Native American } \\ \text { White/Caucasian American } & \text { Other (Please specify) } \\ \text { Bi-racial (please identify) } & \end{array}$




\section{Appendix D}

\section{Wilderness Questionnaire Script and Administration Directions}

\section{Directions: Please read over the complete sheet of directions before administering the questionnaires.}

These questionnaires must be administered by someone other than the three counselors of the course (i.e. course logistician or other TON employee).

Gather the group of students in an area free of distractions and away from their staff members. Make sure that the students are separated far enough from each other that they cannot observe the other's answers or distract one another. Continue to observe them throughout the complete administration. Hand out one stapled set of questionnaires and a writing utensil to each of the students.

Once the above has been completed and the students are ready to answer the questionnaires, read the following script aloud to them:

"What I am about to read is on the front page of your questionnaire. Please read along with me as I read it aloud.

The following questionnaire will be used for research purposes only. It is voluntary and will in no way affect your performance ratings. No one other than the researcher and his university committee members will view your completed questionnaire. Neither the staff members nor your referring agent will have access to how you have answered the questionnaire.

The first two pages request information about you. The following three pages are questionnaires that ask questions about the leaders of your course. Please look at the top of these pages to identify the counselor you are responding to for that particular page. You should complete on page per counselor.

After you have completed the questionnaires, please put them in the addressed tamperproof envelope near me. SHOW THEM THE ENVELOPE Once all of the participants have completed the questionnaires and they have been enclosed in the envelope, the envelope will be sealed. The envelope will be put in the mail as soon as is reasonably possible and will only be opened by the principal investigator.

Please answer the questions to the best of your ability. If you have any questions or concerns, please share them with me.

Does anyone have any questions? (Pause and look around to make sure everyone understands the directions.) Thank you for your help."

When all of the students have competed the questionnaires, please seal the envelope in front of them and put it in a mailbox as soon as reasonably possible.

Thank you for your help,

Dave Cummins 


\section{Appendix E}

\section{Wilderness Counselor Research Questionnaire}

The following questionnaire will be used for research purposes only. It is voluntary and will in no way affect your job or employment. No one other that the researcher will view your completed questionnaire. After you have completed the questionnaires please enclose them in the addressed tamperproof envelope. Once all of the counselors have completed the questionnaires and they have been enclosed in the envelope, the envelope should be sealed. Please answer the questions to the best of your ability. If you have any questions or concerns, please feel free to contact Dave Cummins at (304)-292-6589.

What is your name?

What is your age?

What is your gender?

What race are you? (Please check one)

African American
Asian American
Hispanic/Latino American
Native American
White/Caucasian American
Other (Please specify)

What is the highest degree that you have achieved?

What major or discipline did you focus on?

How long have you been employed at Spectrum Wilderness?

How many wilderness therapy courses have you lead?

What are the total number of days you have worked wilderness therapy courses? 